A us der Chirurgischen Universitätsklinik Zürich. (Direktor: Prof. Dr. P. Clairmont.)

\title{
Ausschaltung der Bauchhöhlensensibilităt durch Blockierung der Nervi splanchnici und der Rami communicantes des lumbalen Grenzstranges.
}

\author{
Von G. A. Preiß, Assistenzarzt der Klinik.
}

(Mit 9 Textfiguren.)

Die Frage der Sensibilität der Bauchhöhle hat in den letzten Jahren große Wandlungen durchgemacht. Sie geklärt zu haben, ist nicht in letzter Linie das Verdienst der zahlreichen Bestrebungen, die von chirurgischer Seite unternommen wurden, auch intraabdominelle Eingriffe in lokaler Anästhesie ausführen zu können.

Nachdem Schleich am ChirurgenkongreB 1892 seine ersten Mitteilungen über die Lokalanästhesie mit Kokain gemacht hatte, teilte er 1894 in seiner Publikation ..Schmerzlose Operationen" mit, daß er 28 La parotomien. darunter 3 Gastro. stomien und 2 Cholecystotomien ganz in örtlicher Betäubung ausgeführt habe.

Nach anfänglich großer Skepsis bürgerte sich seine Methode bald in den meisten Kliniken ein.

I900 schreibt v. Frie dländer aus der A I bertschen Klinik in Wien von 68 in Lokalanästhesie ausgeführten Operationen am Abdomen, darunter von 5 Gastrostomien, 2 Colostomien, 2 Ovariotomien.

$\mathrm{B}$ akes teilt 1904 mit, daß an seiner Station die intraabdominellen Eingriffe entweder in reiner $\mathrm{Schle} \mathrm{ich} \mathrm{scher} \mathrm{Lokalanästhesie}$ oder in kombinierter Narkose ausgeführt werden. Mit Schleich werde das Abdomen eröffnet, in Ätherrausch erfolge die Orientierung und das Zurechtlegen der Organe zum Eingriff, für die Manipulationen am Magen-Darm selbst wurde jede Anästhesie ausgesetzt. Für die Reposition und Peritoncalnaht werde von neuem ein kurzer Ätherrausch eingeleitet und der weitere Schluß der Bauchhöhle in Infiltrationsanästhesie zu Ende geführt.

An der v. Mikuliczschen Klinik wurden bereits vor fast 25 Jahren größere Bauchoperationen in Lokalanästhesie ausgeführt. Got ts te in berichtet aus den Jahren 1896 bis 1898 von 71 Eingriffen 
am Magen, wobei in 49 Fällen Narkose, in 22 Fällen Lokalanästhesie angewendet wurde, darunter sind auffallenderweise von i2 Magenresektionen 9 in örtlicher Betäubung operiert worden. Wie oft allerdings bei diesen Eingriffen die Narkose zu Hilfe genommen werden mußte, geht aus der Publikation Got ts te in s nicht hervor. Er betont aber ausdrücklich, wie viel leichter selbst dekrepide $\mathrm{Pa}$ tienten solch große Eingriffe von mehreren Stunden Dauer in Lokalanästhesie aushalten als Patienten, die in Narkose operiert werden. Diese Erkenntnis macht die zahlreichen Bestrebungen verständlich, die in der Folge zur Verbesserung der lokalen Anästhesierungsmethoden für intraabdominelle Eingriffe und zur Klärung der sensiblen Versorgung der Bauchhöhle unternommen wurden.

Len na nder (I90I) kam auf Grund seiner Untersuchungen zu dem Schluß, daß sämtliche Organe, die nach Abgabe des Nervus recurrens vom Vagus oder Sympathicus innerviert werden, keinen Schmerz-, Druck-, Wärme;, und Kältesinn haben, ebensowenig seien Mesenterien und Netz empfindlich. In hohem Grade empfindlich ist nach ihm nur das Peritoneum parietale. Die Schmerzen beim Zug an den Mesenterien kommen zustande durch Reizung der das Peritoneum parietale der hinteren Bauchwand innervierenden Interkostal- und Lumbalmerven des cerebrospinalen Systems, während dem sympathischen System jede sensible Reizleitung abgesprochen wird.

Die Untersuchungsergebnisse Le n n a nders sind im Laufe der Jahre teils auf experimentellem Wege, teils durch klinische Beobachtungen revidiert worden.

$\mathrm{Kast}$ und $\mathrm{Meltz}$ er fanden auf Grund ihrer Tierversuche eine Empfindlichkeit der normalen Organe, die bei entzündeten Organen erheblich gesteigert war.

Wilms kommt zu der Überzeugung, daß der ganze Darmtraktus als solcher empfindungslos, daß dagegen die Mesenterien vermittels feiner, mit den mesenterialen Gefäßen verlaufender spinaler Nervenfasern Schmerzempfindung vermitteln können.

Entgegen dieser Ansicht glaubt $R$ itter nach Experimenten am Hund an die Schmerzempfindlichkeit aller Organe. Die von den meisten Chirurgen und von ihm selbst bei Laparotomien in Lokalanästhesie beobachtete Schmerzunempfindlichkeit der Organe selbst erklärt er nicht durch Allgemeinwirkung des Anästhetikums, sondern durch den trocknenden Einfluß der Luft auf die Darmoberfläche und dadurch bedingte Lähmung der feinsten sensiblen Nervenendigungen.

Propping, der die Versuche Ritters wiederholte, betont 
starke Schmerzempfindung bei der Unterbindung der Mesenterialgefäße.

F instere r kommt auf Grund seiner Erfahrungen bei Laparotomien in Lokalanästhesie zu der Überzeugung, daß alle Manipulationen am Magen und Darm schmerzlos sind, wenn jede, auch die geringste Zerrung an den Mesenterien vermieden werden kann. In der Bauchdeckenspannung sieht er den sichersten Indikator für die Schmerzempfindung. Solange der Patient nicht spannt, ist ein gewisses monotones, mehr prophylaktisches Klagen ohne Bedeutung.

Rittershaus und Hesse (I9IO-I9II) sahen konstant Schmerzhaftigkeit bei Hantierungen am Mesocöcum und am Mesenteriolum.

Das Postulat des adäquaten Reizes von Nothnagel aufgestellt, von L. R. Müller, Goldscheider und Neumann verfochten, wurde später wieder fallen gelassen und noch in letzter Zeit teilt A. W. M e yer (1919) in Heidelberg aut Grund von Experi. menten an Katzen mit, daß Magen und Darm einschließlich der sie bedeckenden Serosa auf adäquate und nichtadäquate Reize schmerzunempfindlich seien, dagegen die Mesenterien bis zum Muskelschlauch des Magens und Darmes für alle Reize Schmerzempfindung besitzen.

I9ı2 schreibt M üller in seiner histologischen Studie über die Darminnervation, daß nach der Ansicht sämtlicher Autoren der Splanchnicus sensible Nerven enthalte. Die bei den tabischen Darmkrisen auftretenden Schmerzen seien ein weiterer Hinweis auf die sensiblen Fasern dieser Nerven, die - - in Übereinstimmung mit den histologischen Untersuchungen Langleys - über die Spinalganglien und die hinteren Wurzeln in das Rückenmark eintreten.

Durch die Untersuchungen von $\mathrm{B}$ u e r g e r und $\mathrm{Ch} \mathrm{urchmann}$ (1915) aus der Garrèschen Klinik wurde festgelegt, daß Reizung der abdominellen Ganglien sowie der Nervi splanchnici von starker Schmerzäußerung gefolgt war.

$M$ ülle r vergleicht die Leibschmerzen mit den Schmerzen, die bei Angina pectoris oder bei Gallenstein- oder Nierenkolik auttreten. Ihr Charakter ist vollständig verschieden von der Art der Schmerzen, die bei Reizung rein spinaler Nerven zustande kommen. Während letztere lokalisiert sind, springen Reizzustände der visceralen Nerven leicht auf das ganze vegetative Nervensystem über und können sich in vasomotorischen Störungen wie Blässe des Gesichts, meßbarer Blutdruckerhöhung oder gar in Speichelfluß und SchweiBausbruch dokumentieren. Steigern sich diese visceralen Schmerzen, so können sie älınlich wie die Herzschmerzen das Gefühl des Unerträglichen oder der Vernichtung hervorrufen. B raun schlägt deshalb vor, diesen prinzipiellen Unterschied auch durch den Sprach- 
gebratuch zu kennzeichnen und nennt diese Empfindungen ,abdominelle Sensationen".

Die Untersuchungen von $\mathrm{Kapp}$ is an Hunden und die etwa zu gleicher Zeit, aber unabhängig von ihm vorgenommenen Experimente $\mathrm{N}$ e u m a n $\mathrm{n}$ brachten zum erstenmal eine befriedigende Erklärung der sensiblen Versorgung der Bauchorgane. Beide Autoren kommen zu denselben Resultaten, die K a p p is wie folgt zusammenfaßt und die, wie er glaubt, mit einiger Vorsicht vom Hund auf den Menschen übertragen werden können.

Praktisch kommt eine Sensibilität der Organe nicht in Betracht, sei es, daß die Nervenfasern schon vor dem Erreichen der Organe aufhören, oder zu fein sind und in zu geringer Zahl in ihnen endigen. Dagegen sind die Mesenterien, das kleine Netz, der Ansatz des großen Netzes am Magen, die Gegend des Choledochus, Cysticus, Hepaticus und der Leberpforte, der Nierenhilus, und die Gegend der großen Gefäße usw. stark schmerzempfincllich, d. h. alle diejenigen Partien, in welchen die sensiblen Nerven noch in genügender Stärke und Anzahl sich vorfinden. Die sensible Reizleitung geschieht auf dem Wege der abdominellen Ganglien, der Nervi splanchnici und der Rami communicantes lumbales, also vermittels des Sympathicus.

La ignel-Levastine schreibt 1903 in seinen Recherches sur le plexus solaire, daß die Sensibilität der Bauchhöhle allein durch den Vagus vermittelt würde und daß dem Sympathicus jeder sensible Einfluß abgehe, während Th. Kochcr schon I 896 auf Grund seiner Beobachtungen an Wirbelsäulen- und Rückenmarksverletzten zur gegenteiligen Ansicht kam und klinisch sicher feststellen konnte, daß über den Vagus keine sensiblen Einflüsse aus der Bauchhöhle zentripetal geleitet werden. Dic klinischen und experimentellen Erfahrungen haben dann auch die Ko ch e r sche Beobachtung bestätigen können und es gilt heute als sicher, daß allein der Sympathicus die sensible Leitung von der Bauchhöhle zum cerebrospinalen Nervensystem vermittelt. Mit dieser Erkenntnis war eigentlich der Weg für die Anästhesierung der Bauchhöhle vorgezeichnet.

$\mathrm{K}$ a p p is und Finsterer haben deshalb $191 \mathrm{I}$. mit Hilte der paravertebralen Anästhesierung versucht durch Ausschaltung der Rami communicantes in Brust- und Lendenteil eine vollständige Anästhesie der Bauchhöhle zu erreichen. Wie zu erwarten war, mußte diese Methode bei guter und sorgfältiger Technik volle Erfolge haben. $\mathrm{S}$ i e g e l nennt sie sogar das normale Betäubungsverfahren auf Grund seiner Erfahrungen an über ıooo Fällen. 
Die Idee stammt eigentlich von Sellheim, der schon 1905 die Interkostal- und Lumbalnerven bei ihrem Austritt aus dem Foramen intervertebrale anging, allerdings damals noch nicht mit der Absicht, die Anästhesie auf die Rami communicantes und den Sympathicus auszudehnen.

I9I I nahm Lac we n mit Erfolg diese Versuche wieder auf und gab der Methode den Namen "paravertebrale Anästhesie".

Zur Vereinfachung der Methode versuchte dann $\mathrm{Kapp}$ is in der Folge an Tierexperimenten die Segmentbezüge der einzelnen Organe zu bestimmen, um die Zahl der nötigen Injektionen und damit auch die Zeit für das Anästhesierungsverfahren zu verringern. Aber auch damit ließen sich keine befriedigenden Resultate erzielen, indem Überkreuzungen der einzelnen Nerven und Grenzüberschreitungen an der Tagesordnung sind.

$\mathrm{Kapp}$ is hat dann das Verfahren selbst als zu kompliziert aufgegeben. 1913 erprobte er ein neues Verfahren und teilte 1914 am deutschen Chirurgenkongre $\beta$ in Berlin mit, er sei daran, eine Methode auszuarbeiten, mittels deren er direkt die Nervi splanchnici von hinten her zublockierentrachte.

I 918 erschien in den Beiträgen zur klinischen Chirurgie W end. lings Arbeir über die Ausschaltung der Nervi splanch$\mathrm{nici}$ durch Leitungsanästhesie, die er durch Injektion durch die Bauchdecke von vorn her erreicht. Er sticht $\mathrm{I} \mathrm{cm}$ unterhalb des Processus xiphoideus und $1 / 2 \mathrm{~cm}$ links der Medianlinie ein und nimmt auf die vorliegenden Organe, Leber, Magen, Pankreas, Colon, keine Rücksicht. Gefahren sind dieser Methode sicherlich nicht abzusprechen, wenn sie auch vielleicht im ersten Moment als übertrieben angesehen werden. Wendling hat nach seinen Angaben mit dieser Anästhesie gute Erfolge gehabt. Er berichtet über 36 Fälle mit nur einem Versager. In einem Falle wurden $10 \mathrm{ccm}$ einer I proz. Novokain-Suprareninlösung in eine Vene injiziert, wobei ein allerdings vorübergehender aber schwerer Collaps eintrat. Die Ansicht

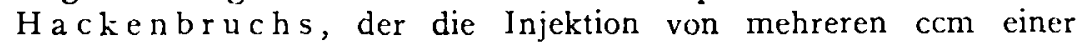
Novokain Suprareninlösung in cine Vene für belanglos hält, besteht dagegen auch nach unseren Erfahrungen wie nach der Ansicht WCndlings nicht zu Recht. Die intravenöse Injektion, die unbe dingt gefährlich ist, kann aber durch vorherige Kontrollaspiration in die Glasspritze sicher vermieden werden. Gegen die Technik Wend lings spricht dieser Zufall nicht. Ein Vorteil seiner Methode besteht vielleicht darin, daß bei der Eröffnung der Bauchhöhle der Stichkana! nachgeprüft - unglücklicherweise bei der Injektion abgebrochene Nadeln leicht entfernt werden können. Das Anstechen von Gefäßen allein - ohne daß in dieselben injiziert würde - braucht nicht gefürchtet $\mathrm{zu}$ werden.

Unscres Wissens ist bis heute die W e n d l ing sche Methode von keiner Seite nachgeahmt worden und auch in der Aarauer Kranken- 
anstalt hat man offenbar das Verfahren aufgegeben, um so mehr, als 1919 die Publikation von $\mathrm{Kapp}$ is erschien, der die Blockade der Splanchnici durch Injektionen vom Rücken her erstrebt, wobei nur Muskulatur, keine Organe durchstochen werden müssen.

Die Technik der Ka p pis schen Splanchnicusanästhesie ist folgende: Der Patient befindet sich in Seitenlage mit kyphotisch gekrümmter Wirbelsäule. Nach Desinf ektion der Haut wird $7 \mathrm{~cm}$ lateral der Medianlinie unterhalb des unteren I2. Rippenrandes eine kleine Hautquaddel gebildet, dann die Injektionsnadel in einem Winkel von ca. 30 Grad zur Sagittalebene an dieser Stelle eingeführt. Nach 6 bis $8 \mathrm{~cm}$ stößt man an die Seite des Wirbelkörpers, welchem entlang die Nadel wcitergeführt wird, bis die Spitze frei ist, sie befindet sich dann sicher am Übergang der Seiten- in die Vorderfläche, d. h. dort, wo die Splanchnici und der Grenzstrang verlaufen. Dasselbe wird auf der andern Seite wiederholt. Injiziert werden je 20 bis $40 \mathrm{ccm}$ einer I proz. Novokain-Suprareninlösung. Damit sind die Nervi splanchnici beiderseits unterbrochen. Zur Ausschaltung des lumbalen Anteils der visceralen Sensibilität empfiehlt $\mathrm{K}$ a p $p$ is das nämliche Vorgehen wie unter der 12. Rippe auch unterhalb der Höhe des ersten Lendenwirbel-Querfortsatzes beiderseits zu wiederholen und hier noch je ro bis $20 \mathrm{ccm}$ derselben Lösung zu injizieren.

Die Erfolge, die Kappis mit seiner Technik an über 200 Fällen erreicht hat, forderten zur Annahme der Methode auf. Wir haben deshalb im Sommer Igrg das Verfahren an der Züricher Klinik eingeführt und bis heute 192 Fälle in Splanchnicusanästhesie operiert. Trotz klarer Schilderung der Technik in der Arbeit von $\mathrm{Kap}$ p is hatten wir das Bedürfnis, die Injektionen nicht gleich am Lebenden auszuführen, sondern Vorversuche mit Farbstoffeinspritzungen (Methylenblau) an der Leiche anzustellen. Außerdem habe ich im Anatomischen Institut (Prof. Felix) an einer erwachsenen männlichen Leiche den ganzen Sympathicus von den Spinalnerven ausgehend bis zu den makroskopisch noch darstellbaren peripheren Ausläufern auspräpariert.

Das sympathische, vegetative, viscerale oder nach Langley autonome Nervensystem, welches die unwillkürlich tätigen Organe versorgt, zerfällt sowohl anatomisch als auch physiologisch und pharmakologisch in zwei große Untergruppen:

I. Das kranialbulbäre und sakra la u to n o meSystem, später (IọI I) von Langley parasympathisches System benannt. 
Seine Fasern stammen aus dem Mittelhirn, dem Bulbus und dem sakralen Teil des Rückenmarks. Aus dem Mittelhirn gelangen die parasympathischen Nervenfasern durch den Nervus oculomo. torius zu ihren Erfolgsorganen, zu M. sphincter pupillae und M. ciliaris, aus der Medulla oblongata oder dem Bulbus durch den Nervus facialis, intermedius, glossopharyngeus und vagus zu den Tränen- und Speicheldrüsen, den Drüsen des Mund-, Nasen- und Rachenraumes, zu Trachea, Lungen, Herz, Leber, Pankreas, Niere, Magen, Dün- und proximalem Dickdarm, aus dem obern Sakralteil durch den Nervus pelvicus zu distalem Colon, Rectum, Blase und Genitalien.

2. Das eigentliche sympathische oder thorakal-

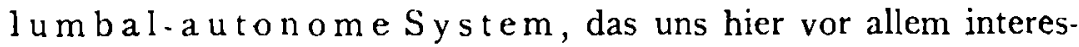
siert, bezieht seine Fasern aus dem thorakalen und lumbalen Teil des Rückenmarks. Von ihm soll in der Folge hauptsächlich die Rede sein.

Für das gesamte vegetative Nervensystem ist $\mathrm{e}$ ine $\mathrm{ge}$ meinsame pharmakologische Reaktion charakteristisch, nämlich die Wirkung des Nikotins. Ebenso charakteristisch ist, daß die vegetativen Nerven im Gegensatz zu den Fasern des animalen Systems niemals direkt vom Zentralorgan $z u$ ihren Erfolgsorganen ziehen, sondern sich zunächst in einem Ganglion aufsplittern und von hier aus als neues Neuron zu den peripheren Organen verlaufen. Diese Umschaltung kann entweder in den Ganglien des Truncus sympathicus (Grenzstrang oder Stammstrangi, den sog. vertebralen Ganglien oder erst in den prävertebralen Ganglien (z. B. Ganglion coeliacum, Ganglia mesenterica usw.) stattfinden. An diesen Ganglien oder Umschaltstellen greift das Nikotin an, und zwar im Sinne einer vollständigen Lähmung nach einer anfänglichen Reizwirkung.

Die Untergruppen des vegetativen Systems, de r $\mathrm{S}$ y $\mathrm{m} p$ at $\mathrm{h}$ icus und der Parasymathicus sind gewissermaßen pharmakologische Antagonisten. Das sympathische System wird durch Adrenalin erregt, d. h. das Adrenalin verstärkt die Wirkung der sympathischen Fasern, ob diese normal erregend oder hemmend wirken, das parasympathische System wird durch Atropin gelähmt, durch Muskarin, Pilocarpin, Physostigmin und Cholin erregt. Im Gegensatz zum Nikotin, das auf die Ganglien einwirkt, greifen Atropin, Adrenalin, Muskarin usw. die peripheren Endigungen an. 
Außer dem animalen und vegetativen System wird noch ein drittes Nervensystem unterschieden, dem ein noch weitgehenderer Grad von Unabhängigkeit und Selbständigkeit zukommt als dem vegetativen System. Langley nennt es „enteric system" (Auerbachsche und Meißnersche Plexus für Magen- und Darmtraktus). Es ist in den Erfolgsorganen selbst eingebettet.

Das Schema des vegativen Nervensystems in M e yers und Gottliebs Experimenteiler Pharmakologie (S. 136) gibt einen Überblick über Ursprung, Verlauf und periphere Endigung der auto. nomen Nerven. Es ist heute allgemein bekannt und vielfach reproduziert (in B raun, Örtliche Betäubung 1919, S. 27; Villiger, Die periphere Innervation, I9I9, S. 138 und I39 (Sympathicus und Parasympathicus getrennt); Buhre, Die Leitungsanästhesie bei Operationen in der Bauchhöhle, B runs' Beiträge 1919, Bd. II8 I, S. 55; B ircher, Die operative Behandlung der Ulcuskrankheit, Schweiz. Rundschau für Medizin, Heft 24/26, I918, S. 44; C a s tellino und Pende, Patologia del simpatico, 1915, S. 86), weshalb es überflüssig erscheint, dasselbe auch an dieser Stelle nochmals wiederzugeben.

Das sympathische Nervensystem setzt sich zusammen aus den beiderseits der Wirbelsäule verlaufenden Trunci sympathici, den Grenzsträngen, die sich aus einer großen Anzahl von Ganglien, den Grenzstrangganglien, aufbauen, welche untereinander durch die Rami interganglionares in Verbindung stehen. Im thorakalen und sakralen Teil ist die Anordnung der Grenzstrangganglien streng segmental - jedem Interkostalnerven entspricht im allgemeinen ein Ganglion - während im Halsteil und, wie wir weiter unter noch sehen werden, auch im Lumbalteil diese Metameric nicht besteht.

Die acht Halsganglien sind zu drei oder sogar zu zwei zusammenge:chmolzen, dem Ganglion cervicale superius, medium und inferius. Vom ersteren setzt sich die Pars cephalica des Sympathicus kopfwärts fort.

Die. Grenzstränge verlaufen im Hals- und obern Brustteil lateral der Wirbelkörper, konvergieren im untern Brustteil, indem sie - entsprechend ihrem Durchtritt durch das Zwerchfell lateral vom Splanchnicus minor - mehr ventral und medial verlaufen Im Lumbalteil behalten die Grenzstränge ihre mehr mediale Lage bei, sie liegen auf der Vorderfläche der Lendenwirbel, divergieren im Sakralteil wieder, wo sie medial der Foramina sacralia anteriora verlaufen. Gegen das Os coccygis vereinigen sich beide im Ganglion impar und bilden so eine nach unten konvexe Schlinge die Ansa 
sacralis. Mit dem Zentralnervensystem, dem Rückenmark, steht der Grenzstrang durch die Rami communicantes in Verbindung.

Langley, dessen Forschungen wir in der Hauptsache die Klärung des sympathischen Faserverlaufs verdanken, hat dafür folgendes Schema entworfen (Fig. I).

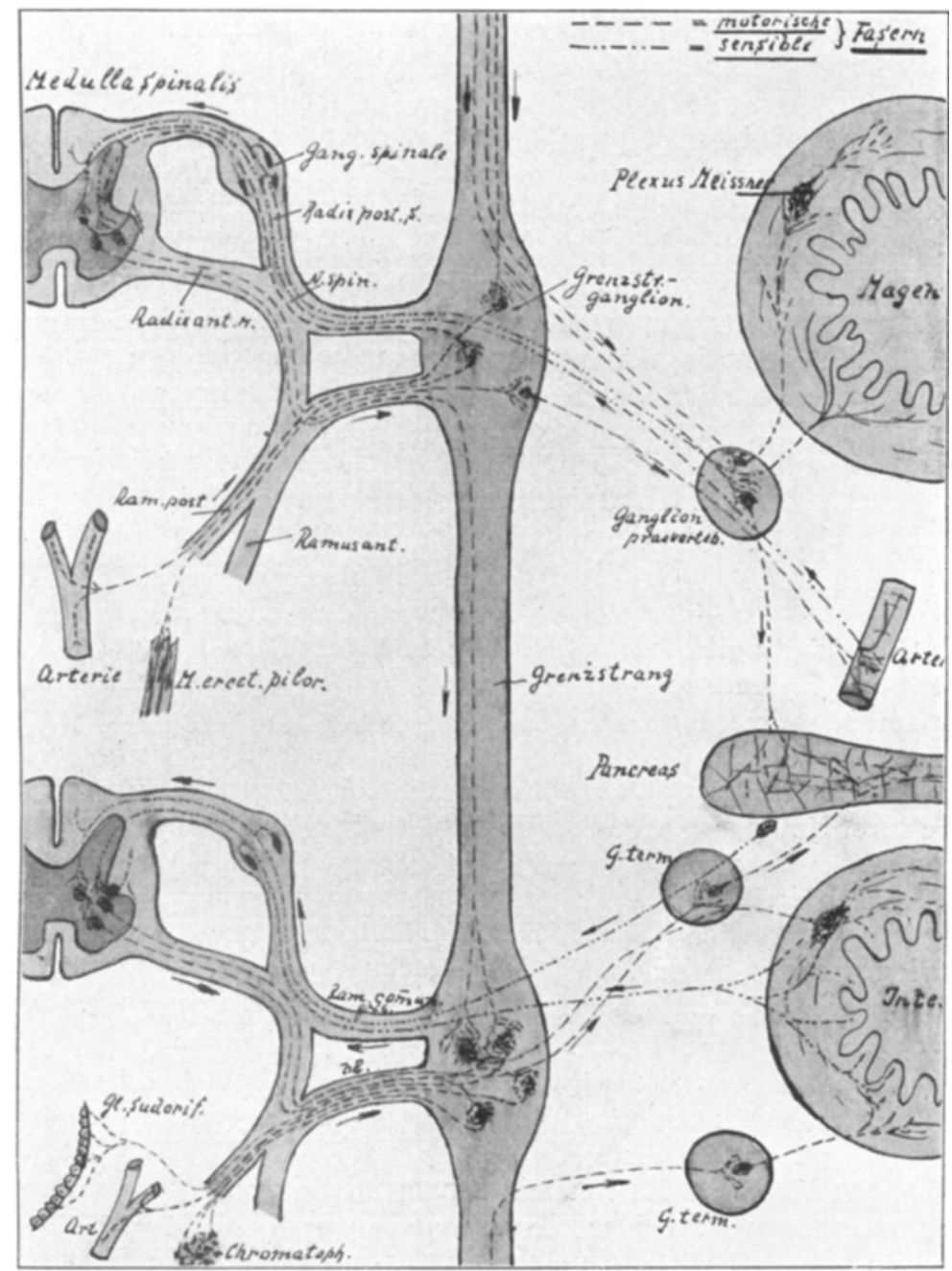

Fig. 1. Schema des sympathischen Faserverlaufes nach Langley und di Cajal. tus Castellino und Pende, "Patologia del simpatico" S. 27. Motorische Fasern gestrichelt, sensible strichpunktiert. Rechts vom Grenzstrang Endigungen zu den Eingeweiden. I inks vom Grenzstrang Endigungen zur Haut. 
Die sympathischen Fasern nehmen ihren Ursprung in der als Tractus intermediolateralis bezeichneten Zellsäule des ganzen Thorakal- und obern Lendenmarks, $d$. $h$. in den dorsalen Partien der lateralen und der Basis der hinteren Säulen. Zwischen Medulla spinalis und der Peripherie sind nach Langley eingeschaltet :

1. Zwei motorische oder efferente Neurone (gestrichelt).

2. Ein sensitives oder afferentes Neuron (strichpunktiert).

I. Das efferente, spino-fugale Neuron setzt sich zusammen aus:

a) dem markhaltigen präganglionären Neuron,

b) den marklosen postganglionären Neuron,

also aus mindestens zwei, oft aber auch aus drei und mehr Neuronen, welche sich in den Grenzstrangganglien (den vertebralen Ganglien) oder den prävertebralen Ganglien (Plexus coeliacus, Mesentericus sup. usw.) aufsplittern.

Diese postganglionären, grauen, marklosen, nach L a ngley eigentlich sympathischen Fasern haben keinen interganglionären Konnex untereinander, sondern ziehen in der größten Zahl von den Grenzstrangganglien rückläufig durch die Rami communicantes grisei, oft separat, oft mit den Rami communicantes albi vereint zu den korrespondierenden Spinalnerven und erreichen mit ihnen die Organe der Haut. Zum kleinen Teil ziehen sie zu den prävertebralen Ganglien und von dort zu den innern Eingeweiden. (Vgl. Fig. I und Schema Meyer und Gottlieb).

Die präganglionären Fasern können sich, wie wir oben gesehen haben, entweder in den Grenzstrangganglien oder in den peripheren, prävertebralen Ganglien aufsplittern. Es ist für das Verständnis der Reflexvorgänge ferner von Wichtigkeit, daß eine und dieselbe präganglionäre Faser auf ihrem ganzen Verlaut mit Hilfe ihrer Kollateralen und Endigungen mit mehreren sympathischen Zellen in Beziehung treten kann, so daß Impulse, die von einer Stelle des Rückenmarks auf sie übergehen, auf eine ganze Anzahl postganglionärer, sympathischer Neurone übertragen werden können. Ga skell nennt die Elemente, welche die Übertragung eines Reizes auf mehrere sympathische Neurone vermitteln „connector elements".

2. Das afferente, sensitive oder spinopetale Neuron, stark in der Minderzahl, durchzieht als eine Faser die sympathischen Nerven von der Medulla bis zur Peripherie. Ihr trophisches Zentrum liegt im Ganglion spinale. Es hat mit den sympathischen Ganglien keine Beziehungen, sondern durchzieht dieselben einfach. Es ist fraglich, ob diese afferenten Bahnen überhaupt sympathische Elemente sind, oder ob sie einfach dem cerebrospinalen System angehören. Sicher sind nach Langley sämtliche sympathische Zellen motorische, nicht sensible Zellen. Die motorischen Neurone gelangen durch die 
Rami communicantes aibi zum Grenzstrangganglion, die sensitiven Fasern durch die Rami communicantes grisei.

Besteht diese Doktrin von Langley zu Recht, so sind Reflexvorgänge im sympathischen System nur.mit Vermittlung der Medulla möglich. Die Langleysche Theorie hat aber auch ihre Anfechter gefunden. So glauben Dogiel, LaignelLevastine, Michailow, Müller u. a. Autoren an die Existenz zweier afferenter Neurone, die ebenso wie die motorischen sich in eine post- und präganglionäre Faser teilen (siehe Fig. 2).

Nach ihnen vollziehen sich demnach die Reflexe nicht nur mit Intervention der Medulla spinalis, sondern können auch ohne dieselbe mit Hilfe der Grenzstrangganglien vor sich gehen. In dieser Richtung hin unternommene Experimente haben tatsächlich gezeigt, da $B$ bei Tieren, denen man das Lumbalmark zerstört hat, alle vegetativen Funktionen weiter persistieren, wenn auch weniger energisch als bei normalen Tieren. Luciani sagt: „Nei animali privati del midollo, esiste un equilibrio assai grande della salute e della vita." Wenn also auch in normalem Zustand die sympathischen Ganglien ihre Impulse von höher gelegenen Zentren des Rückenmarks und des Gehirns erhalten, so sind sie doch auch imstande, ohne diese Impulse stctig regulierend auf die Organe des vegetativen Lebens einzuwirken. Villige $r$ hat in der neuesten Auflage seiner "Peripheren Innervation" (S. 152) als Beispiel einer sympathischen Innervation die Versorgung der Blase dargestellt, aut die ich hier speziell verweisen möchte.

Wenn auch, wie oben dargestellt, die Impulse einer und derselben präganglionären Faser auf mehrere Segmente übertragen werden können, so besteht doch, wie namentlich $\mathrm{L}$ angley und van Rynberk nachgewiesen haben, eine gewisse, wenn auch nicht strenge Metamerie des sympathischen Nervensystems. Auch $\mathrm{K}$ a p p is kommt bei seinen Versuchen, die Segmentbezüge der einzelnen visceralen Organe zu bestimmen, zu denselben Schlüssen, wenn er auch selbst zugibt, daß eine strenge Segmentierung nicht besteht, sondern daß vielfach Überkreuzungen und Grenzüberschreitunger vorkommen. Klinisch von Wichtigkeit ist diese Metamerie geworden durch die $\mathrm{Headschen} \mathrm{hyperalget} \mathrm{is} \mathrm{chen}$ Zonen und den Boasschen paravertebralen Druckpunkt. Es wird sogar angegeben, daß die Hy peralgesie gewisser Hautbezirke schon bestehen kann, bevor eigentliche viscerale Schmerzen auftreten. 
Mit der Erkenntnis sensibler Bahnen im sympathischen Nervensystem war es noch nötig, genau über die topographisch-anatomische Lage der sympathischen Nerven orientiert zu sein, um mit

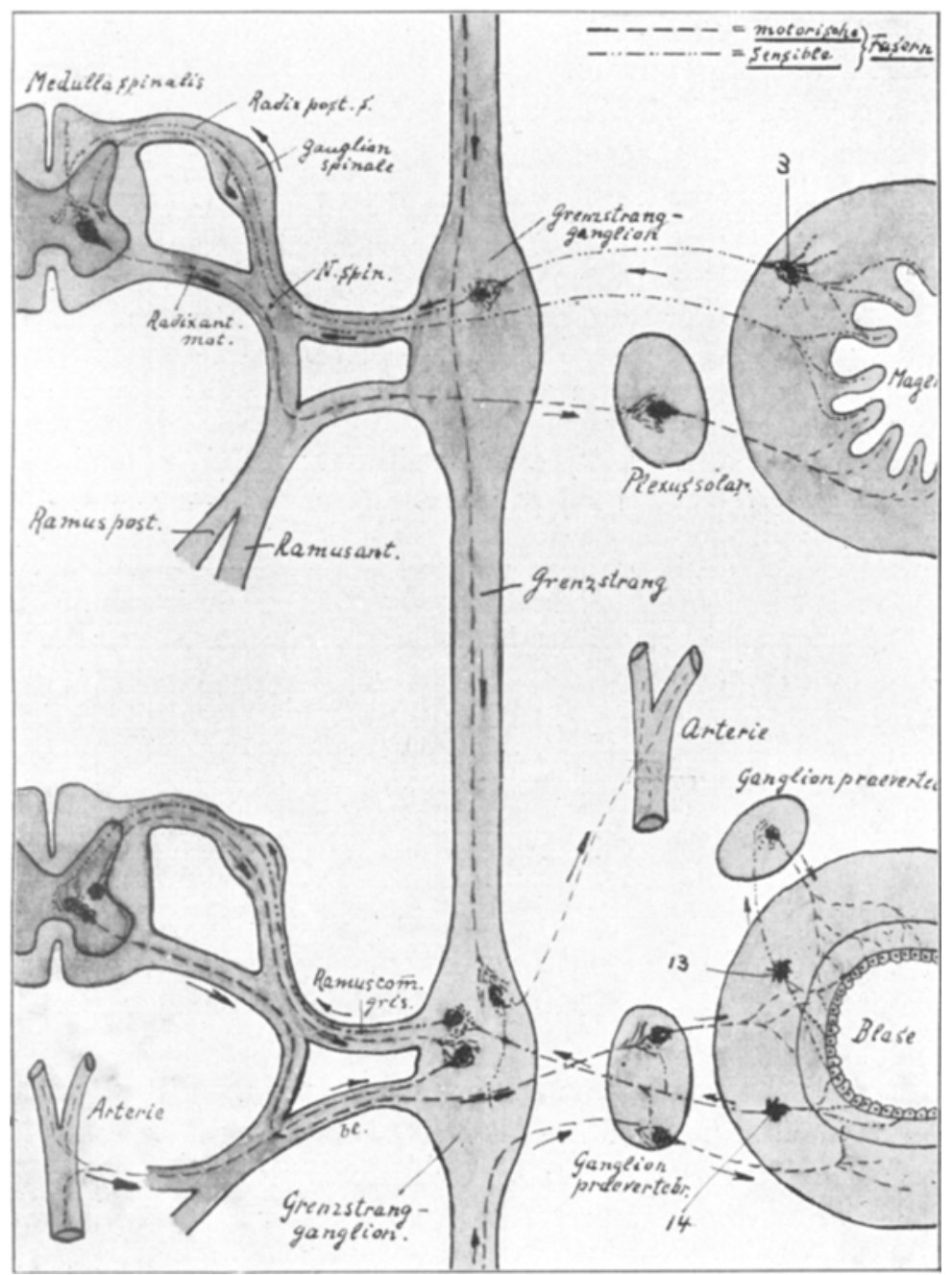

Fig. 2. Schema des sympathischen Faserverlaufes nach Laignel-Levastine und Dogiel. Aus Castellino und Pende, "Patologia del simpatico" S. 37. Motorische Fasern gestrichelt, sensible strichpunktiert. 3,13 und 14 sind sensible periphere Fasern, durch welche die sympathischen Reflexe ohne Vermittlung der Medulla zustande komnen. 
Hilfe der lokalen Anästhesie die viscerale Sensibilität auszuschalten.

Aus dem anatomischen Atlas von $\mathrm{Hirschfeld}$ und Leveillé entnehme ich folgende Tafeln, welche die Topographie des sympathischen Nervensystems und der abdominellen Plexus sehr schön zur Darstellung bringen.

Fig. 3 gibt einen Überblick über das ganze sympathische Nervensystem der rechten Körperhälfte. Die inneren Organe sind nach links hinübergezogen, rechte Lunge, rechte Niere und die rechts gelegenen Partien des Dünn- und Dickdarms sind entfernt. Man überblickt die ganze Ganglienkette des Grenzstranges, ihren Zusammenhang mit den Nerven des Cerebrospinalsystems und ihre, peripheren Endigungen. Man beachte die streng segmentäre Anordnung der Grenzstranganglien im Lumbalteil, die im Widerspruch steht zu den Befunden an unseren Leichen.

Fig. 4 zeigt die Anordnung der abdominellen Plexus und ihre Beziehungen zu den großen Gefäßen. Mit Ausnahme der rechten Niere und eines kleinen kardialen Magenteils sind sämtliche Baucheingeweide entfernt. Zu beiden Seiten der Wirbelkörper laufen die Grenzstrangganglienketten. Man sieht den Durchtritt der rechten Nervi spanchnici durch das Zwerchfell, ferner ihre innigen $\mathrm{Be}$ ziehungen zum $\mathrm{N}$. vagus. Dargestellt sind außerdem die Verbindungen der Grenzstrangganglien mit dem Plexus aortico-lumbalis und den Plexus mesentericus superior und inferior.

Fig. 5 stellt den Plexus solaris und die benachbarten Plexus dar und ihre Beziehungen zu den umliegenden Organen. Der Magen ist an der Cardia durchschnitten und bis zur Pars horiz. sup. duod. entfernt. Die dem Plexus solaris direkt aufliegende Partie des Pankreas ist herausgeschnitten, der ganze Dünndarm von der Flexura duod. jejunalis an und der Dickdarm sind entfernt. Der Leberstiel ist auspräpariert. Über die Flexura duodeno-jejunalis ziehen die Art. mesenterica sup. und sie begleitend der gleichnamige sympathische Plexus.

Nach Test ut verlaufen die sympathischen Nerven im thorakalen und lumbalen Teil nach folgendem Schema: 


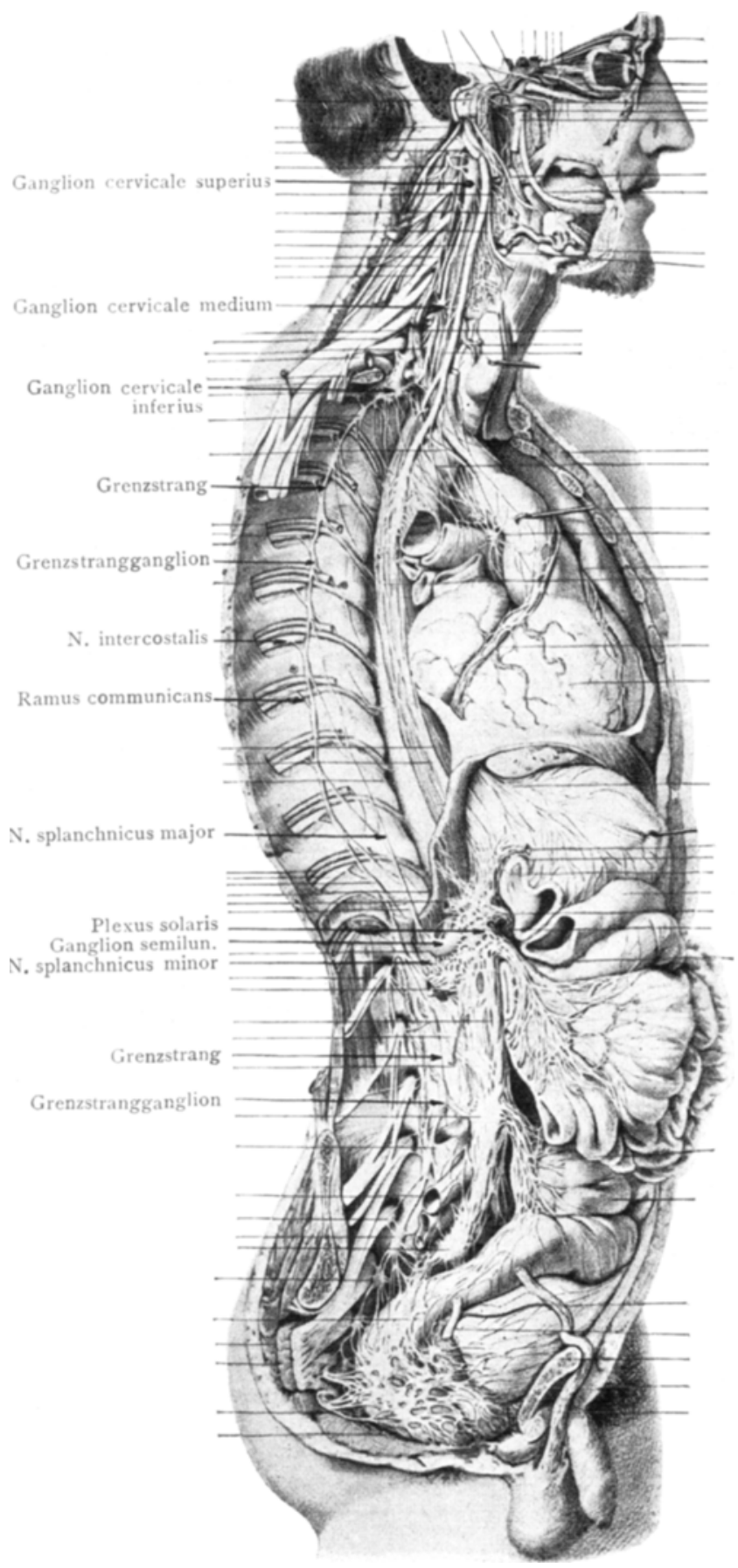

Fig. 3. Sympathisches Nervensystem. Aus Hirschfe'd und Leveillé, Tafel 73 und 74 


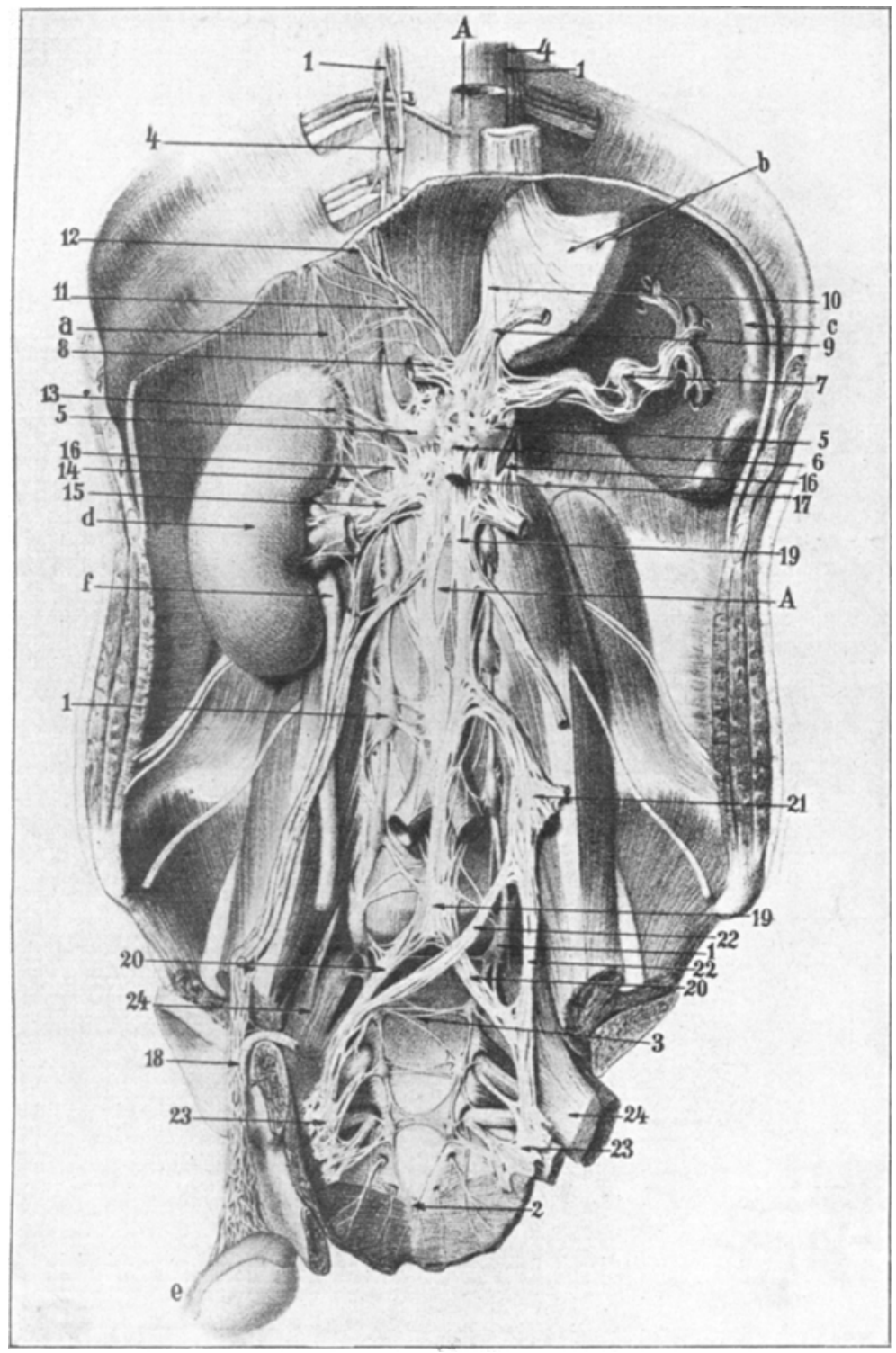

Fig. 4. Die abdominalen sympathischen Plexus. Aus Hirschfeld und Leveillé, Taf. 69. A Aorta, a Diaphragma, b Magen, c Milz, d Niere mit Capsula suprarenalis, e Testis und Epididymis, f Ureter. I Grenzstrangganglien, 2 Ganglion impar, 3 Anastomosen der beiden Grenzstränge, 4 Splanchnicus major, 5-5 Ganglia semilunaria, 6 Plexus solare, 7 Pl. splenicus, 8 PI. hepaticus, 9 Pl. coronarius ventr., so Vagusäste, II Pl. diaphrag. und Capsularis sup., 12 Äste des N. phrenicus, 13 Pl. suprarenalis, 14 Pl. capsularis inf., $15 \mathrm{Pl}$. renalis, 16 Splanchnicus minor, i7 Pl. mesent. sup., $18 \mathrm{Pl}$. spermaticus, $19 \mathrm{Pl}$. lumboaorticus, 20 Teilung desselben, 2 I Pl. mesent. inf., 22-22 Seine Anastomosen mit 23-23 Pl. hypogastricus, 24-24 Sacrale Plexus. 
I. Thorakaler Teil:

a) Rami externi = marklose graue Fasern in geringer Zahl, die mit den Arteriae intercostales zur Peripherie ziehen.

b) Rami interni

$$
\begin{aligned}
& \text { Fila ossea } \\
& \text { F. cardiaca } \\
& \text { F. oesophagea } \\
& \text { F. aortica } \\
& \text { F. pulmonalia }
\end{aligned}
$$

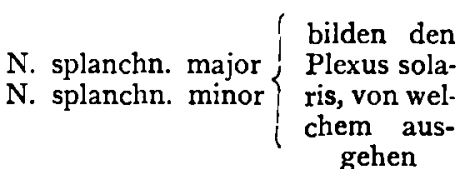

Pl. diaphr. inf.

Pl. gastricus

$\mathrm{Pl}$. hepaticus

Pl. splenicus

Pl. venae port.

Pl. mes. sup.

Pl. suprarenalis

$\mathrm{Pl}$. renalis

Pl. spermaticus

2. Lumbaler Teil:

a) Rami externi = marklose graue Fasern in geringer Zahl, mit den Arteriae lumbales zur Peripherie ziehend.
b) Rami interni $\left\{\begin{array}{l}\text { bilden den Plexus } \\ \text { aorticusabdom.,von } \\ \text { welchem ausgehen }\end{array}\right.$
F. ossea
F. anastomo- $V$. cava inf.
F. vascularia $\underset{\text { für }}{\text { tica }}$
A. mesent. inf.
A. lumbales
A. iliaca communis
u. ihre Äste

Der Nervus splanchnicus major nimmt seinen Ursprung mit vier oder fünf Wurzeln aus dem 6., 7., 8. und 9. Grenzstrangganglion des thorakalen Stammstranges, er kann aber auch schon vom 5. Ganglion thorakale Fasern beziehen, of t aber auch noch vom Io. Nach Vereinigung der einzelnen Fasern zieht er etwa in der Stärke eines Streichholzes medialwärts, nach rorn und distal direkt den Wirbelkörpern und Zwischenbandscheiben aufliegend und von der Pleura bedeckt, durchdringt das Zwerchfell zwischen Crus mediale und Crus intermedium, rechts mit der Vena azygos, links mit der Vena hemiazygos, und ergießt sich, nach medial umbiegend, beiderseits in die Ganglia semilunaria. Oberhalb des Durchtritts durch das Zwerchfell beschreibt A rnold ein Ganglion splanchnicum.

Der Splanchnicus minor entspringt mit zwei bis drei Wurzeln aus dem 10., II., oft auch dem 12. thorakalen Grenzstrangganglion, zieht meist isoliert, oft auch mit dem major vereinigt zur Bauchhöhle. Er liegt bei seinem Durchtritt durch das Diaphragma lateral vom Splanchnicus major, zwischen diesem und dem Grenzstrang. Auch er biegt nach der medialen Seite um und begibt sich mit einem kleineren Teil der Fasern zum Ganglion semilunare, mit dem größten Teil zum Plexus renalis und suprarenalis. Unterhalb seines Durchtritts durch das Zwerchfell hat $\mathrm{Cru}$ veilhier ein kleines Ganglion splanchnicum beschrieben. 
Der Ramus renalis des Splanchnicus minor kann selbständig aus dem I2. thorakalen Ganglion entspringen und heißt dann N e rvus splanchnicus imus.

Die für den Sympathicus beschriebenen Variationen sind in keinem bis jetzt bekannten Fall derart, daß dadurch ein Versagen der Anästhesie eintreten müßte.

$\mathrm{H}$ a lle r beschreibt einen Grenzstrang, der etwa in der Höhe der 6. Rippe aufhörte und sich etwas unterhalb der 7. Rippe neu

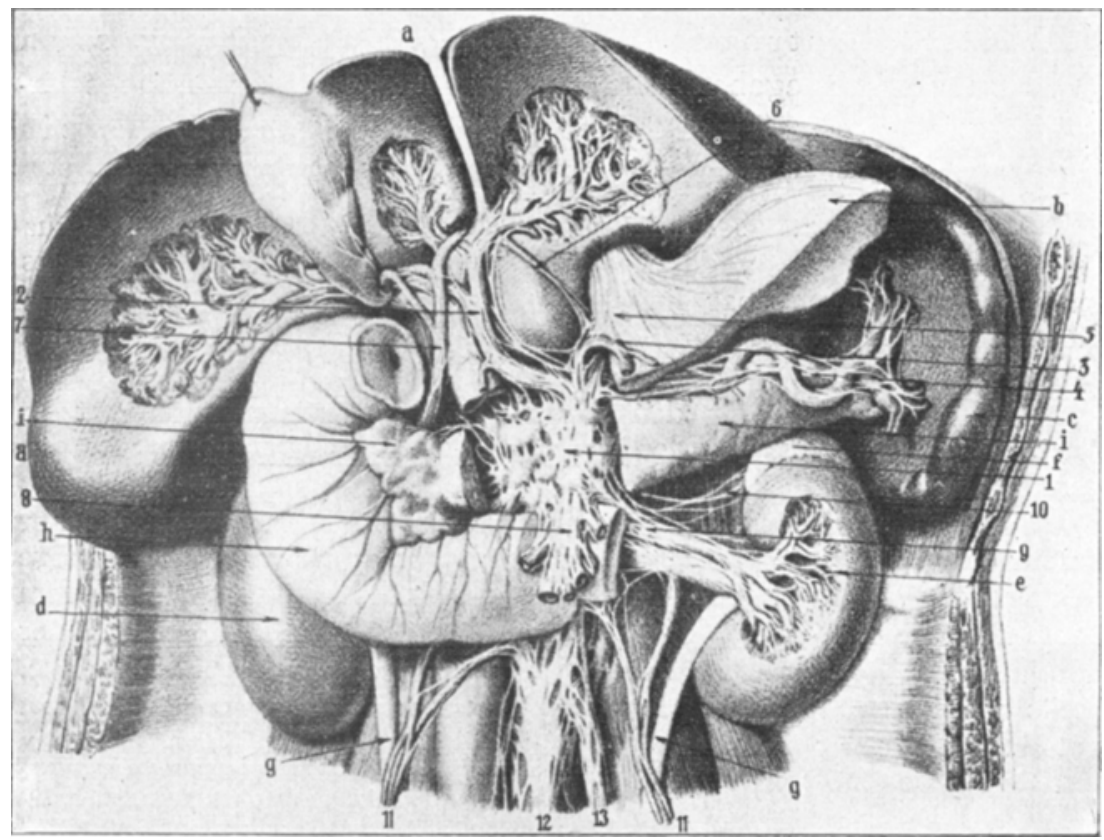

Fig. 5.

Der Plexus solaris und seine sekundären Plexus. Aus Hirschfeld und Leveillé, Taf. 7o. a Leber, b Magen, c Milz, d-e Nieren, f Capsula suprarenalis, g-g Ureteren, h Duodenum, i-i Pankreas, durchtrennt zur Darstellung des Plexus solaris. I Plexus solaris, 2 Plexus hepaticus, 3 Plexus coronarius ventriculi, 4 Plexus splenicus, 5 Anastomosen des Vagus mit dem Plexus solaris und Plexus coronarius, 6 Äste des Vagus zur Leber, 7 Plexus der Gallengänge, 8 Ursprung des Plexus mesentericus superior, 9 Plexus renalis, Io Plexus capsularis, I I-I I Plexus spermaticus, I 2 Plexus aorticus abdominalis, 13 Teil des Plexus mesentericus inferior.

bildete. Analog dazu sah Bichat eine Unterbrechung des Grenzstranges zwischen dem thorakalen und dem lumbalen Teil. Nach 
Lobstein können die Splanchnici majores beiderseits durch die Aortenöffnung des Zwerchfells in die Bauchhöhle eintreten, $\mathrm{K} \circ \mathrm{ll}$. ma n n sah in sehr seltenen Fällen einen Nervus splanchnicus superior, der seine Fasern einmal aus dem Plexus cardiacus, einmal aus den Grenzstrangganglien des Halses und ein drittes Mal aus dem obersten Thorakalganglion bezog.

Dis Lage der Splanchnici beiderseits ist nicht symmetrisch zur Medianlinie, sondern sie liegen, entsprechend ihrer Endigung. im Plexus coeliacus, der die Arteria coeliaca ringförmig umgibt, in leichter Sinistroposition zur Medianlinie, wie schon Wendling angegeben hat. Diese Verschiebung ist meines Erachtens auch für die Technik der Anästhesie nach $\mathrm{Kapp}$ is nicht ganz ohne Bedeutung.

Beide Ganglia semilunaria bilden zusammen den Plexus coeliacus. Das linke, das meist weniger umfangreich ist als das rechte, liegt unmittelbar links der Arteria coeliaca der Aorta aut, das rechte liegt nach der Seite zu der Vena cava, nach oben der Leber und ventral dem Kopf des Pankreas auf. Der Plexus coeliacus umgibt also ringförmig den Tripus Halleri, welcher konstant in der Höhe des untern Randes des 12. Brust- oder oberen Randes des ersten Lendenwirbels aus der Aorta abdominalis entspringt. Die Gestalt des Plexus coeliacus ist ziemlich variabel. Oft imponiert er mehr als einheitliches Gebilde, oft scheint er in mehrere selbständige Ganglien gegliedert. Der radiäre Verlauf der zahlreichen aus ihm entspringenden Fasern hat zu dem Namen Plexus solaris oder Sonnengeflecht geführt. Von ihm aus gehen folgende teils paarige, teils unpaarige Plexus, von welchem dann die Nervenfasern meist in Begleitung der entsprechenden Arterien und ihren Verzweigungen zu den Erfolgsorganen ziehen.

I. Paarige sekundäre Geflechte des Plexus coeliacus:

a) Plexus phrenicus

b) Plexus suprarenalis

c) Plexus renalis

d) Plexus spermaticus oder ovaricus.

2. Unpaarige Greflechte:

a) Plexus gastricus superior

b) Plexus hepaticus

c) Plexus lienalis

d) Plexus mesentericus superior.

Die Endverzweigungen des letzteren stehen in ganzer Ausdehnung des Darmkanals in Verbindung mit dem Plexus myentericus oder Auerbachschem Geflecht und dem Plexus submucosus oder Meißnerschem Geflecht. 
Nach unten setzt sich der Plexus coeliacus fort in den Plexus aorticus abdominalis, dieser in den Plexus mesentericus inferior, den Plexus hypogastricus superior und den Plexus iliacus.

Versorgt wird der Plexus coeliacus und seine sekundären Geflechte durch:

1. die Nervi splanchnici

2. die abdominalen Äste der beiden Vagi.

3. mehrere direkte A ste aus den 1 umbalen Grenzstrang. g a nglien.

Die Vagi haben, wie wir heute sicher wissen, mit der Sensibilität der Abdominalorgane nichts zu tun. Ihre anatomischen Beziehungen zu den Ganglia semilunaria und den Nervi splanchnici durch die Ansa Wrisbergi hat Testut auf S. 269 seiner Anatomie humaine dargestellt.

Die Sensibilität der Bauchhöhle kann also nur durch die Splanchnici und die Äste aus den lumbalen Grenzstrangganglien vermittelt werden. Hier hat die Blockierung einzusetzen, um chirurgische Eingriffe im Abdomen schmerzlos ausführen zu können.

Für die Splanchnici hat $\mathrm{Kapp}$ is den Weg, wie oben beschrieben, genau angegeben, allerdings muß hier erwähnt werden, $\mathrm{da} B$ wir je nach dem Alter, der Größe und dem Bau des $\mathrm{Pa}$ tienten die Einstichstellen etwas variieren, auch gehen wir rechterseits, infolge der mehr ventralen Lage der Splanchnici mit dem Einstich etwas mehr lateral, während die linke Nadel steiler geführt wird. Ist der Thorax stark ptotisch, d. h. fallen die Rippen steil ab, so wählt man mit Vorteil den oberen Rand der 12. Rippe oder führt die Nadeln leicht kopfwärts geneigt ein. Dabei sind Durchstechungen der Lunge nie vorgekommen, außer in einem Fall im Anfang unserer Versuche, wo die Nadel, unterhalb der I2. Rippe eingeführt, nach oben abgewichen sein muß. Klinisch war kein auffallend starker Tiefstand der unteren Lungengrenzen nachweisbar. Bei dem betreffenden Patienten trat sofort im Anschluß an die Injektion Hustenreiz und blutiger Auswurf auf. Letzterer hielt zwei Tage lang an, der übrige Verlauf war komplikationslos.

$\mathrm{Ka} \mathrm{p}$ is hat in seinen neuesten Mitteilungen seine ursprüngliche Methode dahin abgeändert, daß er von einer Einstichstelle beiderseits unterhalb der I2. Rippe die Nadel mehr kranial führt, offenbar in demselben Bestreben wie wir, die Splanchnici clamit sicherer an ihrem Stamm, d. h. vor ihrem Durchtritt durch das 
Zwerchfell zu erreichen, wobei sich die anästhesierende Lösung leicht im retropleuralen, prävertebralen Gewebe ausbreitet.

Vor seinem neuesten Vorschlag, mit der Nadelspitze etwas von Wirbelkörper wegzubleiben, sehe ich keine Vorteile. Ich habc mich an vielen Leichenversuchen, bei denen mit kleinen Mengen Methylenblau injiziert wurde, stets davon äberzeugen

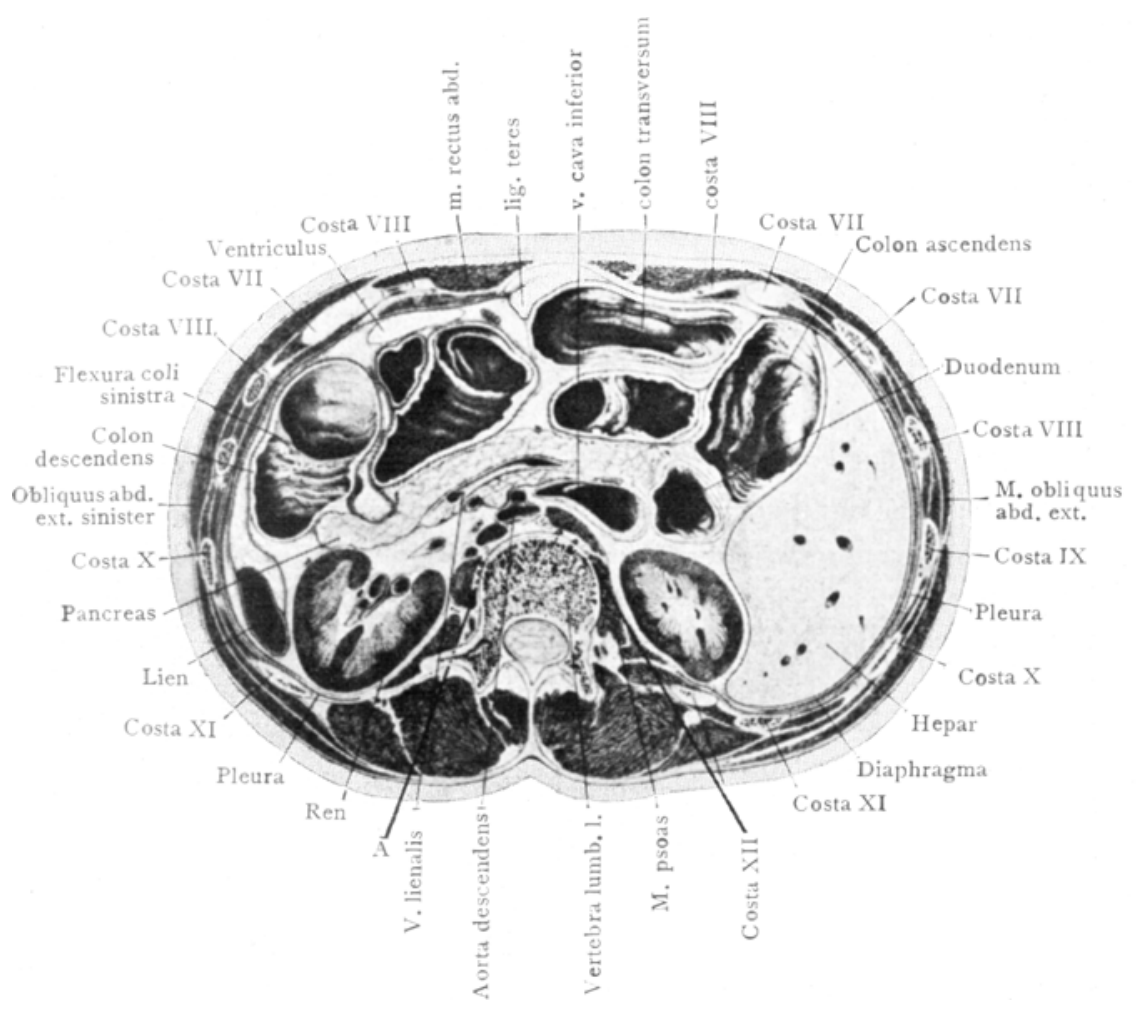

Fig. 6.

Querschnitt durch die Nitte des linken Lendenwirbels nach Braune, Taf. I5. I-I Nervi splanchnici majores, 2-2 Nervi splanchnici minores, 3-3 Trunci sympathici, auf der rechten Seite mehr ventral und medial gelegen. A und B geben die Nadelrichtung an.

können, wie rasch und weit sich die Injektionsflüssigkeit verbreitet, auch wenn die Nadelspitze hart dem Periost auflag.

Fig. 6 zeigt einen Querschnitt durch den menschlichen Kadayer in der Höhe der Mitte des ersten Lendenwirbels, also etwas 
mehr kaudal als die Injektionen tatsächlich vorgenommen werden. Die dem Wirbelkörper direkt aufliegend gezeichneten Kreise I, 2 und 3 stellen die Querschnitte durch den N. splanchnicus major und minor und den Grenzstrang dar. Die Pfeile A und B geben die Injektionsnadeln an. Die linke ist entsprechend der mehr dorsalen und lateralen Lage der Nerven auf dieser Seite weniger steil eingeführt als die rechte. Aus der Figur ist deutlich trsichtlich, wie dic Nadeln lediglich die Rückenmuskulatur und keine Organe durchdringen.

Mit einigen Worten möchte ich noch auf die Interkostalgefäße zu sprechen kommen. Aus statischen Gründen verlaufen sie beiderseits über die konkave Seitenfläche der Wirbelkörper, die Vene stets in Begleitung der Arterie und haben in der Nähe der Wirbelsäule oft ein erstaunlich großes Kaliber. Wenn man auch bezüglich der Gefahr des Anstechens von Gefäßen mit der Injektionsnadel zu ängstlich sein kann, so gibt es immerhin zu denken, daß unsere Nadeln direkt tangential den Interkostalgefäßen geführt werden. Das häufige Anstechen von Gefäßen bei der Splanchnicusanästhesie kommt wohl in der Hauptsache auf Rechnung der Vasa intercostalia und nicht der Aorta oder Cava, dafürspricht a uch, daßman links of tvenöses, rechts of t arterielles Blut aspirieren kann. Sicher lassen sich diese Gefäße aber vermeiden, wenn man für die Injektion die Höhe der I2. Zwischenwirbelscheibe wählt, an die. ser entlang geht, oder wie wir es häufig an Leichen versucht und am Lebenden gemacht haben, die Nadel direkt durch die Knorpelscheibe durchführt. Man spritzt dann, sobald die Nadelspitze frei ist und befindet sich so sicher am richtigen Platz. In den Fig. 7 und 8 ist die Nadel beiderseits durch die 12. Zwischenwirbelscheibe durchgestochen. Bei unseren Leichenversuchen lagen die beiden Splanchnici und der Grenzstrang stets mitten im Farbstoffdepot. Namentlich rechterseits ist diese Art der Injektion wegen der mehr ventralen und medialen Lage der Nerven empfehlenswert. Rückenschmerzen haben die Patienten nach der Operation nicht oder nur ganz vorübergehend bemerkt.

Vorsicht erheischt dieses Verfahren nur bezüglich der Gefahr des Abbrechens von Nadeln infolge des größeren Widerstands 


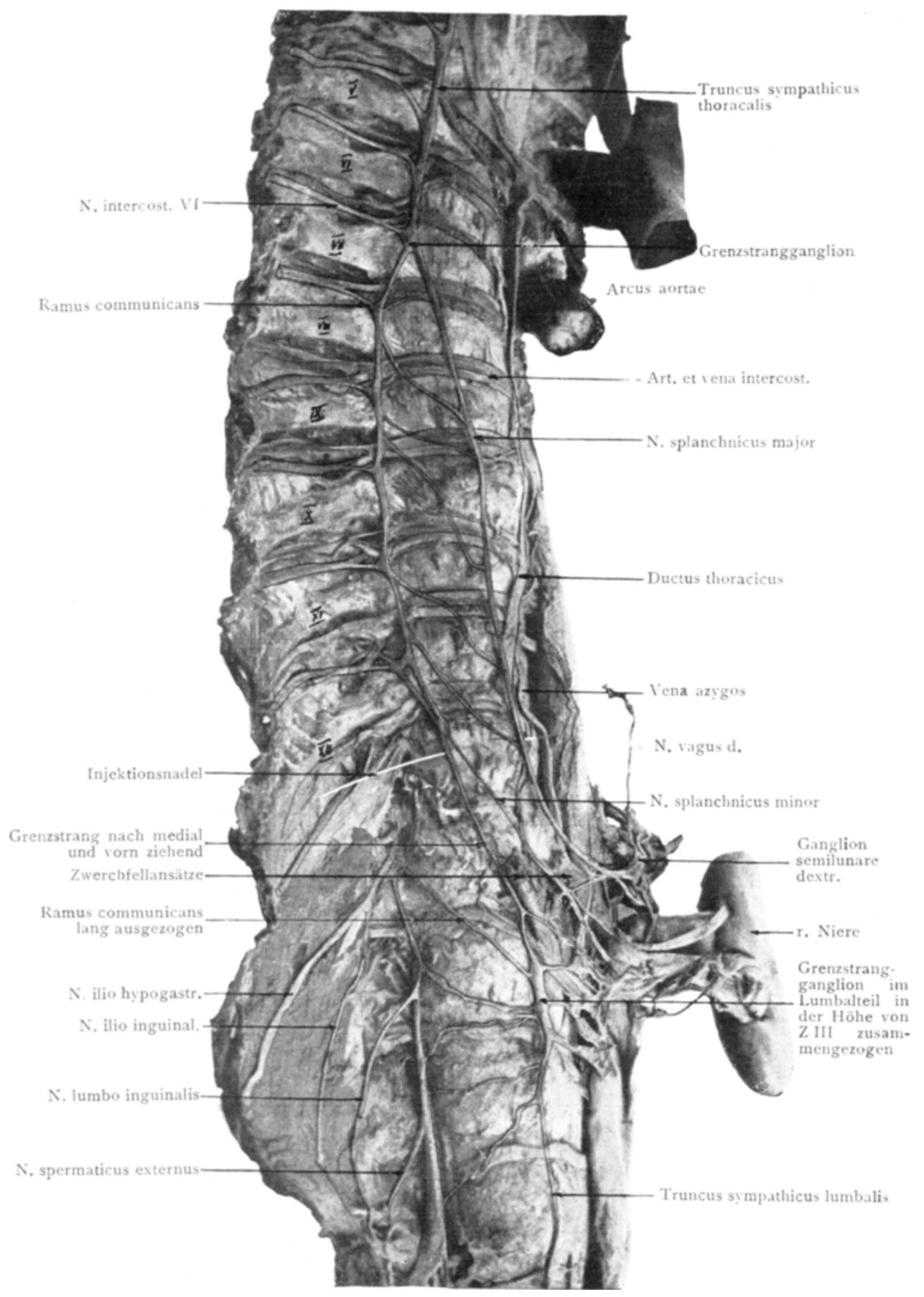

Fig. 7. Truncus sympathicus und Nervi splanchnici der rechten Seite. Eigene I'räparation. Die Injektionsnadel - wie am Lebenden ohne Führung des Auges eingeführt - durchdringt die Fibrocartilago intervertebralis XII. Man beachte die radiäre Anordnung der Rami communicantes in Lumbalteil. 


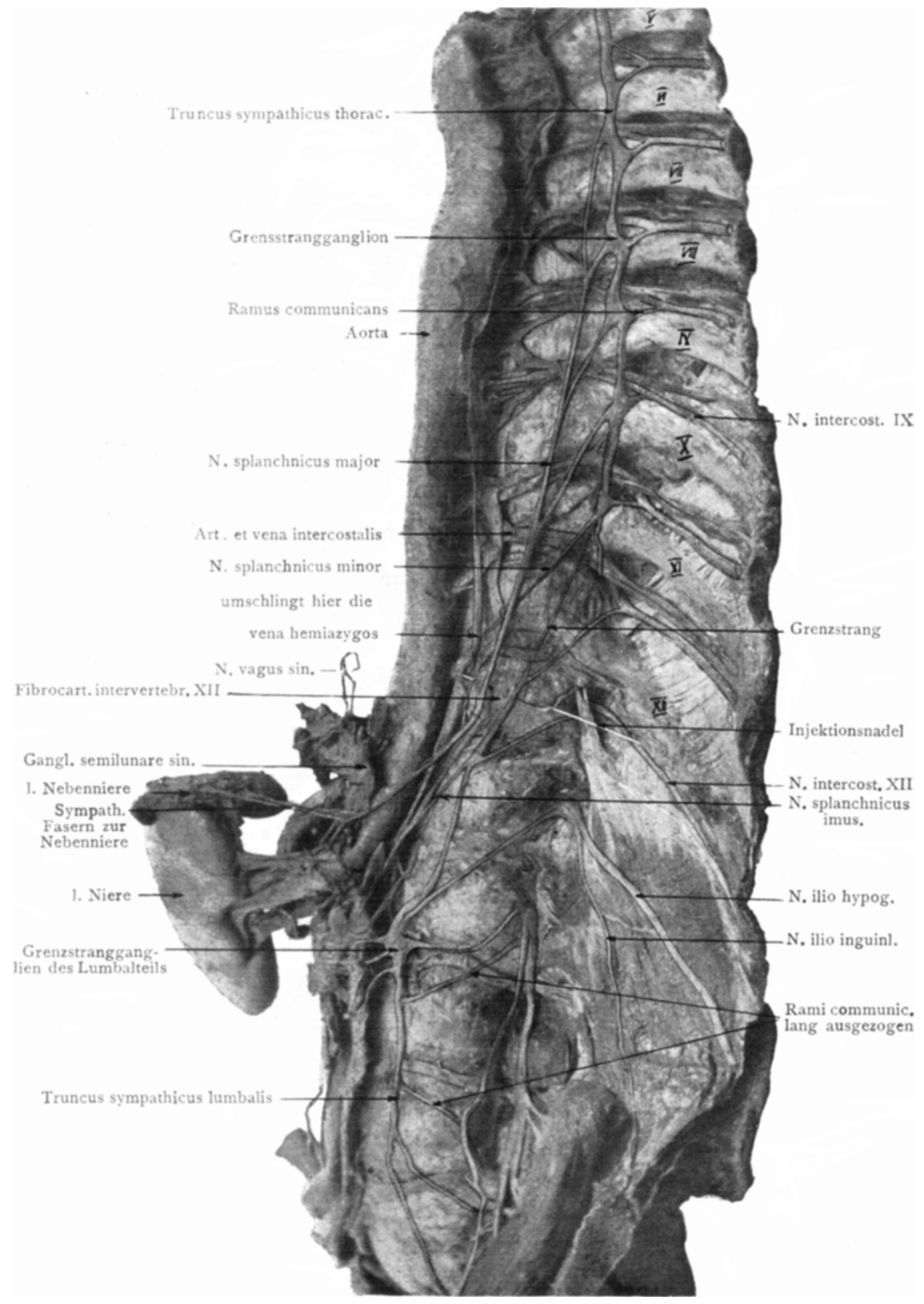

Fig. 8. Truncus sympathicus und Nervi splanchnici der linken Seite. Eigene Präparation. Die Injektionsnadel durchdringt die Fibrocartilago intervertebralis XII. Auch hier Ganglia lumbalia des Grenzstranges zu einem Ganglienknoten in der Höhe von L. III zusammengezogen, auf welchen die lang ausgezogenen Rami communicantes radiär zusammenziehen.

Deutsche Zeitschrift f. Chirurgie, 159 . Bd. 
beim Durchstechen des Knorpels. Uns selbst „st dieser Zufall dreimal passiert, wobei aber die Nadeln stets in der Nähe des Ansatzes oder unmittelbar unter der Haut abbrachen, so daß die. Extraktion der Fragmente leicht vorgenommen werden konnte.

Unser Rekordkanülen aus vernickeltem Stahl haben ein Kaliber von $0,75 \mathrm{~mm}$ und eine nutzbare Länge von mindestens I $2 \mathrm{~cm}$. Wenn es auch wünschenswert ist, da $B$ die Dicke der Nadeln diese Maße nicht übersteigt, so läge doch ein großer Vorteil darin, ihnen zur besseren und leichteren Führung eine größere Starrheit zu verleihen. Vielleicht läßt sich dies mit den aus Tantal gearbeiteten Kanülen erreichen, die allerdings im Preise sehr teuer sind.

Für die Ausschaltung des lumbalen Anteils der visceralen Sensibilität, vermittelt durch Faserzüge aus den lumbalen Grenzstrangganglien, gibt $\mathrm{K}$ a p $\mathrm{p}$ is ursprünglich an, $3 \mathrm{~cm}$ unterhalb der I2. Rippe die Injektion beiderseits $z u$ wiederholen, nach seinen neuesten Mitteilung aber führt er von der gleichen Einstichstelle unterhalb der I 2. Rippe die Nadel einfach kaudalwärts geneigt nochmals ein und legt hier kleine Depots an.

Meine Präparationen und Versuche an der Leiche haben mich aber zu der Überzeugung gebracht, da $\beta$ weder durch das Einführen der Nadel $3 \mathrm{~cm}$ unterhalb der 12. Rippe noch durch ein Neigen derselben in kaudaler Richtung die Unterbrechung der lumbalen Sensibilität gesichert ist.

Wie aus den Fig. 7 und 8 hervorgeht, ist die Anordnung der Grenzstrangganglien im lumbalen Teil keineswegs segmental wie im Thorakalteil. Ich habe sie fast immer auf eine kurze Strecke zusammengedrängt, oft sogar in einen einheitlichen Ganglienknoten vereinigt gefunden. Wenn auch zugegeben werden muß, daß ihr Verhalten zahlreichen Variationen unterworfen ist, worauf auch $\mathrm{Gege} n \mathrm{~b}$ a ur hinweist, so war doch auffallend, da $B$ fast bei sämtlichen Leichen, die ich im pathologischen und im anatomischen Institut zu Gesicht bekam, diese Zusammenziehung oder Vereinigung der lumbalen Grenzstrangganglien deutlich ausgesprochen war. Nicht an allen Präparaten ist die Verschmelzung eine so deutliche wie dies aus Fig. 7 und 8 ersichtlich ist. Oft sind tatsächlich 4-5 Ganglien vorhanden, das erste und letzte 
aber sehr klein und verkümmert, dafür die mittleren um so stärker - man ist versucht zu sagen kompensatorisch - entwickelt. Dieser Afflux von Ganglien findet sich fast konstant in der Höhe des 3. Lendenwirbels und der 3. Lendenwirbelzwischenscheibe und entsprechend diesem Verhalten sind die infolge der medialen und ventralen Lage des lumbalen Grenzstrangs lang ausgezogenen Rami communicantes nicht mehr parallel, sondern radiär angeordnet, laufen oben kaudal, in der Mitte horizontal, unterı kranialwärts auf diese Ganglienverschmelzung zu. Diese gibt dann ihrerseits wieder ziemlich dicke Fasern an den Plexus aorticus lumbalis und die höher gelegenen Plexus ab, namentlich ließen sich oft ansehnliche Faserzüge zu den Nieren, dem Colon ascendens und descendens verfolgen.

Wollen wir also auch hier anästhesieren, so müssen wir mit den unteren Einstichen tiefer gehen als dies $\mathrm{K}$ a p p is vorschlägt.

Bei mittelgroßen Menschen beträgt die Distanz vom oberen Einstich bis zur Höhe des 3 . Lendenwirbels ca. $8 \mathrm{~cm}$. Ich habe auch in dieser Richtung einige Versuche an der Leiche vorgenommen. Durchsticht man die 3. Zwischenwirbelscheibe und injiziert, sobald die Nadelspitze frei ist, so erhält man im Retroperitoneum ein Farbstoffdepot, das immer wieder durch seine weitgehende Verteilung auffällt, meist vom 2. Lendenwirbel bis zum Promontorium hinunterreicht und in seiner Mitte vom lumbalen Grenzstrang und den beschriebenen Ganglienknoten durchzogen wird. Die Injektion ist an clieser Stelle ebenso ungefährlich wie oben, wenn die gleiche Distanz des Hauteinstichs von der Spinallinie beibehalten wird. Es können so keine Organe verletzt werden und die Nadel durchdringt lediglich Muskulatur. Näher der Medianlinie einzugehen ist nicht ratsam, da man dann den Plexus lumbalis anstechen könnte, der sich in dieser Höhe eng an dic Basis des Lendenwirbelkörpers anschmiegt.

Fig. 9 stellt einen Querschnitt durch die 3. Lendenzwischen. wirbelscheibe dar.

Am Lebenden habe ich bis heute diese tiefen Einstiche dreimal ausgeführt. In zwei Fällen (Appendicitis und Ileus infolge Tumor der Flexura lienalis war die Wirkung der Anästhesie eine ausgezeichnete, im dritten Fall, bei dem einer 60jährigen Patientin wegen eines Carcinoma pylori die Resektion des Pylorus und 
Magens nach Mikulicz-Krönlein ausgeführt wurde, war die Wirkung weniger günstig und es mußte am Ende des Eingriffs für den Schluß der Bauchhöhle eine kurze Äthernarkose eingeleitet werden. Bei den zwei ersterwähnten Patienten wurden pro Nadel je $20 \mathrm{ccm}$ einer 2 proz. Lösung, bei der dritten Patientin je $25 \mathrm{ccm}$ einer I proz. Lösung injiziert.

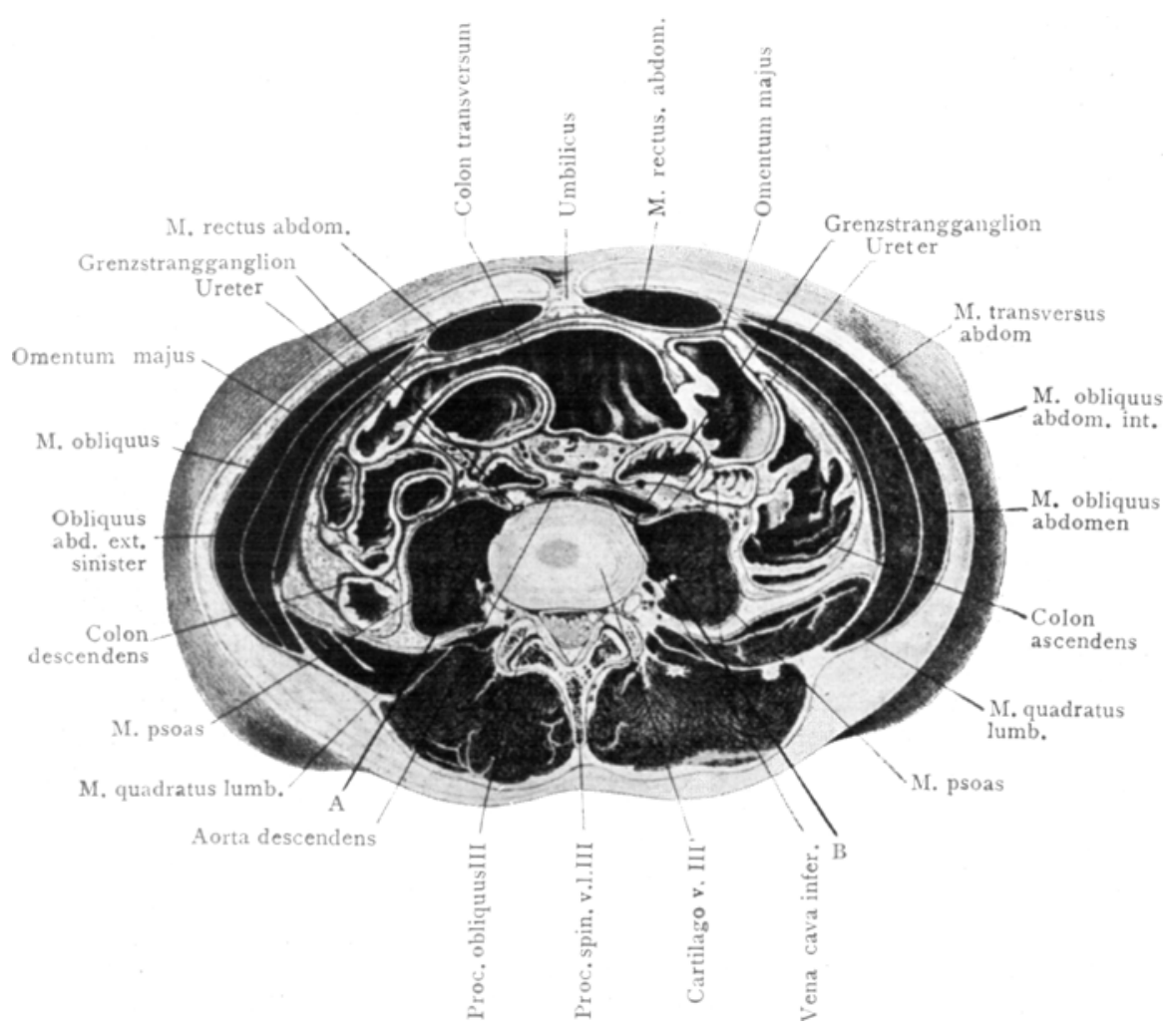

Fig. 9.

Querschnitt unterhalb des 3. Lendenwirbels nach Braune, Taf. 16.

$A$ und $B$ geben die Nadelrichtung an.

Wir müssen allerdings auf Grund unserer Erfahrungen mit der Splanchnicusanästhesie mit nur einer Injektion auf beiden Seiten unterhalb der I2. Rippe und nach den Mitteilungen von $\mathrm{Na}$ a geli und H of $\mathrm{fmann}$, die im gleichen Sinne von der ursprünglichen $\mathrm{K}$ a p p is schen Methode abwichen, sagen, daß es auch so oft gelingt, eine vollkommen befriedigende Anästhesie 
im Ober- und Mittelbauch zu erzielen. Wohl vor allem deshalb, weil - wie schon mehrfach betont - die Ausbreitung der anästhesierenden Flüssigkeit im prävertebralen lockeren Bindegewebe eine überraschend gute ist, und dann auch deshalb, weil wohl die Hauptsache der abdominellen Sensibilität durch die Splanchnici und nur ein verhältnismäßig geringer Teil durch die Fasern aus den lumbalen Grenzstrangganglien vermittelt wird. Dennoch möchten wirfürgewisse Operationen die unteren Injektionen nicht missen, namentlich für Eingriffe an den Nieren, der Milzund dem Colon. Wir blockieren die Splanchnici ja oberhalb ihres Durchtritts durch das Diaphragma, welches nach unten hin der Verbreitung der anästhesierenden Lösung immerhin einige Schranken bietet.

Ist bei gewissen Fällen, z. B. Ileuskranken, eine sichere Diagnosestellung ante operationem bezüglich der Höhe der Okklusion nicht möglich, so ließe sich ev. die Splanchnicusanästhesie kombinieren mit der Lumbal-oder einer der sakralen Anästhesierungsmethoden, wodurch die Sensibilität vom Oberbauch bis ins kleine Becken hinunter ausgeschaltet werden könnte.

An unserer Klinik wurde z. B. letztes Jahr eine Patientin in Splanchnicusanästhesie operiert, die wegen Ileuserscheinungen eingeliefert und bei der sich erst während der Operation als Ursache des Hindernisses eine stielgedrehte Ovarialcyste feststellen ließ. Während die Eventration der Dünndärme und ihre Reposition schmerzlos waren, mußte für die Exstirpation der stark entzündlichen Ovarialcyste eine vorübergehende Narkose eingeleitet werden. Einen zweiten Fall, der sich ev. für das vorgeschlagene kombinierte Verfahren eignen würde, haben wir bis heute nicht mehr gesehen.

Außerordentlich wichtig für die Durchführung der ganzen Operation ohne Narkose ist in allen Fällen eine weite und exakte Umspritzung der Bauchdecken bis zum parietalen Peritoneum für den Laparotomieschnitt. Wird hier gesündigt, so rächt sich dies unerbittlich beim Einsetzen der Haken und beim Abstopfen der Bauchhöhle mit Gazestreifen.

Bezüglich der Konzentration und der Zusammensetzung der 
zu injizierenden Lösungen gehen die Ansichten der verschiedenen Autoren weit auseinander.

$\mathrm{Ka}$ p p is injizierte ursprünglich eine I proz. Novokain-Suprareninlösung in einer Menge bis $120 \mathrm{ccm}$. Neuerdings verwendet er nur noch $1 / 2$ proz. Lösungen, von welchen er bis $100 \mathrm{ccm}$ injiziert.

Wendling spritzte bis $80 \mathrm{ccm}$ einer I proz. Novokain-Suprareninlösung, $\mathrm{N}$ a e g eli bis $70 \mathrm{ccm}$ derselben Lösung. D en k verfährt genau nach den $\mathrm{Kappisschen} \mathrm{Vorschriften,} \mathrm{B}$ uhre verwendet bis $100 \mathrm{ccm}$ derselben Lösung, $\mathrm{D}$ r ün e $\mathrm{r}$ nur $1 / 4 \mathrm{proz}$. Lösung in sehr geringer Menge, so da $B$ er pro Patient kaum auf $0,5 \mathrm{~g}$ Novokain in Substanz kommt. Hof $\mathrm{fmann}$ injiziert bis $100 \mathrm{ccm}$ der folgenden $1 / 2$ proz. Lösung:

Novokain 0,5, Solutio Kal. sulfur. (2 Proz.) 20,0, Solutio Natr. chlorat. (0,9 Proz.) ad 100,0, Sol. suprarenini hydrochlor. (I prom.) guttae XII.

Wir haben, um kleinere Mengen injizieren zu müssen, die Konzentration der Lösung auf 2 Proz. gesteigert und je nach dem Alter und entsprechend dem Körpergewicht gewöhnlich 20-60, selte! einmal $80 \mathrm{ccm}$ folgender Lösung injiziert:

Novokain 2,0, Aqua destillata 100,0, Suprarenin 0,002, Natrium chlorat. 0,7 , Kalium sulfuricum 0,4 .

Es wurden so 2,0 bis maximal $2,35 \mathrm{~g}$ Novokain in Substanz injiziert, ohne daß dabei die Dosen von $K$ appis und $S$ iegel von 3,3 und 3,0 g Novokain bei ihren paravertebralen Anästhesien erreicht worden wären, die von Jurasz für zu hoch und für zu gefährlich für den Pat. gehalten werden. Trotz dieser großen Menge von Novokain, welche die von den Höchster Farbwerken angegebene Maximaldose von $0,5 \mathrm{~g}$ um ein Vielfaches übersteig und trotz der hohen Konzentration der Lösung haben alle Pat. die Injektionen ohne irgendwelche Intoxikations- oder Kollapserscheinungen überstanden. Auch die bei den meisten Pat. Inehrfach gemachten Kontrollen des Urins nach der Operation $\epsilon$ rgaben nie Zeichen von Nierenschädigungen. Immerhin haben wir die Absicht, bei unseren späteren Fällen die Konzentration versuchsweise herabzusetzen, in der Hoffnung, daß es mit zunehmender Technik auch mit verdünnterer Lösung gelingen werde, eine vollständige Anästhesie zu erzeugen, ohne daß quantitativ mehr injiziert werden müßte. Den Zusatz von Kalium sulfuricum werden wir, da wir diesbezüglich nur Gutes gesehen haben, beibehalten. 
Zeitweise trat bei unseren Kranken leichte Blässe des Gesichts, zeitweise Brechreiz, öfters Erbrechen während der Operation auf. Ein abnormes Steigen oder Sinken des Blutdrucks konnte bei keinem der kontrollierten Fälle beobachtet werden, er zeigte, wie dies auch die anderen Autoren hervorheben, ein sehr ungleiches Verhalten. Auch konnten wir bei keinem Patienten eine auffallende Blutfülle des Abdomens oder auffallende Anämie des Gehirns konstatieren.

Wahrscheinlich trifft B u h re mit seiner Ansicht das Richtige, wenn er vermutet, daß die lähmende Wirkung der Anästhesie auf den Splanchnicus wenigstens zum Teil durch das mitinjizierte Adrenalin paralysiert wird, das bekanntlich erregend auf die peripheren sympathischen Endigungen wirkt.

Bei einem großen Teil unserer Patienten, nämlich bei fast $2 / 3$ der Fälle, haben wir jede Vorbereitung mit einem Narkotikum ante operationem weggelassen. Erstens konnten wir uns über die durch Splanchnicusanästhesie erreichbaren Erfolge ohne vorhergegangene Herabsetzung der Schmerzempfindlichkeit durch Morphium, Pantopon oder Scopolamin besser überzeugen und uns ein objektiveres Urteil über die Methode bilden, zweitens hatten wir den Eindruck, daß die Patienten ohne Narkotikum viel weniger unter unangenehmen Nebenerscheinungen, wie quälendem Durstgefühl, Trockenheit der Mund- und Rachenschleimhäute, Erbrechen und auffallendem Schwitzen, zu leiden hatten. Auch $\mathrm{Sa}$ uerbruch konnte ähnliche unangenehme Nebenwirkungen bei Kombination der Novokainanästhesie mit vorhergegangenen Morphiumgaben beobachten. (Persönliche Mitteilung.)

Für die Injektion selbst ist allerdings eine Herabsetzung der allgemeinen Sensibilität namentlich bei empfindlichen Patienten vorteilhaft.

Unsere übrigen Patienten erhielten nur o,ol Morphium oder höchstens 0,02 Pantopon eine Stunde vor Beginn der Operation. Nie wurden höhere Dosen oder Verbindungen mit Scopolamin angewendet, auch von der Verabreichung von Hypnotica am Abend vor der Operation machten wir keinen Gebrauch.

In neueren Mitteilungen von $\mathrm{Br}$ a un und $\mathrm{Buhre}$ schlagen diese Autoren vor, die Nervi splanchnici und die abdominellen Plexus von vorn her nach eröffneter Bauchhöhle zu infiltrieren. Es wird 
dabei folgendermaßen vorgegangen: Umspritzen der Bauchdecken bis auf das parietale Peritoneum nach Braun. Nach Eröffnung der Bauchhöhle wird die Leber mit einem Spatel hochgezogen, der Magen, wenn nötig, nach links verlagert und dann mit dem tastenden Finger die Vorderfläche des I. Lendenwirbels aufgesucht. Nach Beiseiteschieben der Aorta wird die Nadel auf die Mitte des Lendenwirbelkörpers eingeführt und hier ein Depot von $100 \mathrm{ccm}$ einer $1 / 2$ proz. Novokain-Suprarenin angelegt. Die Unempfindlichkeit der Bauchhöhle soll unmittelbar auf die Injektion eintreten und in allen Fällen von Operation im Oberbauch ein vollständig schmerzfreies Manipulieren gestatten (nach B ra un, Methode der Wahl).

Drüner empfiehlt nach eröfneter Bauchhöhle Injektionen auf die große und kleine Kurvatur des Magens, auf den Tripus Halleri, das Duodenum, den Leberstiel und die Arteriae mesentericae superior et inferior.

Der prinzipielle Unterschied beider Methoden liegt vor allem darin, daß Ka p p is die Splanchnici in ihrem Verlauf unterbricht, während $\mathrm{Braun}$ und $\mathrm{Buhre}$ einerseits mit der anästhesierenden Lösung den Plexus solaris, D rüner andererseits dic: Sensibilität noch weiter peripher ausschaltet.

Trotz den guten Erfolgen, die diese Verfasser mit der Anästhesierungsmethode von vorn erreicht haben, werden wir auch in Zukunft das Ka p p is sche Verfahren mit kleineren Modifikationen beibehalten, in der Ansicht - die übrigens auch $\mathrm{Kapp}$ is teilt - daß es nicht in jedem Falle möglich ist, die Manipulationen bis zur Injektion für den Patienten schmerzfrei zu gestalten. Ich denke dabei namentlich an Fälle mit entzündlichen Verwachsungen, mit Peritonitiden, dann vor allem an Fälle, bei denen die Schnittführung der Bauchdecke für die Operation an einer ganz anderen Stelle liegt als sie für die Injektion nötig wäre. Andererseits hat das $\mathrm{Kap}$ p is sche Verfahren schon deshalb etwas Verlockendes, als Anästhesie und eigentliche Operation zeitlich vollständig getrennt sind. Ein weiterer Umstand ist der, da $B$ diese Verfahren von vorn her für therapeutische $Z_{\text {wecke nicht verwendet werden }}$ können. Dagegen kommen diese Methoden in Form von sekundären Injektionen, wie sie $\mathrm{Kapp}$ is auch schon angegeben hat, bei teilweisen Versagern der Splanchnicusanästhesie in Frage, um so mehr als ja nach D rüner ganz geringe Novokaindosen zur Anästhesie genügen sollen. 
Vor allem aber kann ich D r üners Ansicht der Gefährlichkeit der Einstiche von hinten her nicht teilen und habe vergeblich in der Literatur nach der genügenden Anzahl von warnenden Beispielen gesucht. Die Gefahr der Injektion in Gefäße besteht bei allen Methoden in gleicher Weise und kann bei allen durch Kontrollaspirationen gut vermieden werden. Das Anstechen von Gefäßen ohne Injektion in dieselben ist belanglos. Bei den wenigen Pat. unserer Klinik, die $\mathrm{n}$ a $\mathrm{ch}$ der Operation in Splanch. nicusanästhesie - n i e infolge der A n ä st h esi e - ad exitum kamen, konnten wir nie Hämatome beobachten.

Bis heute haben wir zweimal durch intravenöse Injektion akute Novokainvergiftung in Form von schweren, aber vorübergehenden Kollapsen erlebt.

Im ersten Fall handelte es sich um eine 56 jährige Frau mit Ulcus callosum der hinteren Magenwand in der Nähe der kleinen Kurvatur. Nachdem alle vier Nadeln injektionsbereit waren, wurden zuerst links oben und unten, dann auch rechts oben je $20 \mathrm{ccm}$ der üblichen 2 proz. Lösung injiziert, nachdem man sich vor der Injektion stets überzeugt hatte, da $\beta$ kein Blut aspiriert werden konnte. $\mathrm{Nach}$ der letzten Injektion traten plötzlich alarmierende Erscheinungen auf. Die Nadeln wurden deshalb sofort herausgezogen, die Patientin auf den Rücken gelegt. Das Gesicht ist leichenblaß, die Pupillen starr, die Atmung stockt vollständig, da g e g e $\mathrm{n}$ is $\mathrm{t}$ der Puls ohne wesentliche Veränderung. Nach kurzer Zeit treten klonische Zuckungen in den Extremitäten auf. Patientin ist vollständig bewußtlos. Es wird sofort künstliche Atmung eingeleitet, wobei Muskelstarre der obern Extremitäten auffällt. Nach ca. 8 Min. beginnt die Patientin wieder spontan oberflächlich zu atmen. Der Puls ist andauernd gut. Nach 10 Min. ist die Atmung tiefer, Patientin schaut um sich, die Blässe verschwindet langsam, vom ganzen Vorgang hat sie nicht das Geringste bemerkt. Es waren also rechts oben $20 \mathrm{ccm}$ der 2 proz. Lösung in die Vena cava injiziert worden. Drei Wochen später wurde bei der Patientin, die sich in. zwischen vollständig erholt hatte, die geplante Magenresektion unter Wiederholung der Splanchnicusanästhesie ausgeführt. Sie gab dies. mal ohne Zufälle ein ausgezeichnetes Resultat und die Patientin konnte geheilt aus dem Spital entlassen werden.

Beim zweiten Fall, einer 44 jährigen Frau mit Pyloruscarcinom, traten auf die Injektion vơn $25 \mathrm{ccm}$ derselben Lösung ebenfalls unmittelbar nach der Einspritzung ähnliche Symptome auf, nur daß hier - wie bei dem Fall. den IVendling beschreibt - der Pulskaummehrzufühlen war. Auch hier ging der Kollaps ohne bleibenden Schaden für die Patientin nach ca. 15 Min. vorüber. 
Sie wurde einige Tage später in lokaler Anästhesie laparotomiert, wegen ausgedehnter Metastasen konnte allerdings nur noch eine Ernährungsfistel angelegt und die Frau später in leidlichem Zustand nach Hause entlassen werden.

Über ähnliche schwere Zustände von akuter Novokainintoxikation nach zufälligen Injektionen von Novokainlösung in eine Vene berichtet A. Me yer auf Grund seiner Beobachtungen an 4 Fällen.

Zweimal handelte es sich um Struma-Patienten, bei welchen einmal bei der Umspritzung $5 \mathrm{ccm}$ einer I proz. Lösung, das andere Mal für die paravertebrale Leitungsanästhesie eine unbekannte Menge derselben Lösung in eine Vene gespritzt, zweimal betrat es Patienten mit Oberkiefer-Wangen-Carcinomen, bei welchen in die Fossa pterygopalatina je 5 bis $7 \mathrm{ccm}$ der I proz. Novokainlösung in. travenös injiziert wurden.

Es traten bei sämtlichen 4 Patienten noch während und unmittelbar auf die intravenöse Injektion ganz plötzlich einsetzende Krämpfe von ausgesprochen epileptiformem Charakter auf, ferner Atemstillstand, starke Cyanose und Bewußtlosigkeit. Die Operation konnte trotzdem in allen 4 Fällen zu Ende geführt werden, und zwar gleich nach der Injektion und hie und da bis $3 / 4$ Stunden nachher bei absoluter Schmerzunempfindlichkeit und die Patienten erholten sich ausnahmslos vollständig. Auf die in zwei Fällen beobachtete peripherische Analgesie soll weiter unten noch eingegangen werden. Über das Verhalten des $\mathrm{Pulses}$ fehIen leider nähere Angaben.

Andere Erscheinungen von akuter Novokain-Intoxikation erlebte $\mathrm{M}$ e y er zweimal bei Paravertebral-Anästhesie für Bauchoperationen, bei welchen eine unbestimmte größere Menge einer I proz. Novokainlösung injiziert wurde. In beiden Fällen kam es ohne Krampfanfälle zu plötzlicher, hochgradiger Blässe, Atemstillstand, Kollaps, BewuBtseinsverlust und in einem Fall zu einer Totalanalgesie auch außerhalb des Operationsfeldes, was sich durch Unempfindlichkeit beim Zwicken der Haut an den übrigen Körperpartien mit Kocherklemmen dokumentierte.

Außerdem traten bei zwei Strumapatienten nach subkutaner Injektion von ca. $40 \mathrm{ccm} 2$ proz. Novokainlösung eigentümliche Schlafzustände auf, aus welchen die Patienten wohl auf wiederholten Anruf erwachten, in den sie aber sofort wieder verfielen, sobald man sie in Ruhe lieB.

Über die Wirkung der Injektionen von Lokalanästheticis ins Blut waren schon in früherer Zeit von $\mathrm{Kast}$ und Meltzer (I906), von Ritter (1909 und I9II) und von A. Me yer (I914) Tjerversuche angestellt worden. 
Alle Autoren, die hauptsächlich an Hunden experimentierten, konntın feststellen, daß nach intravenösen Injektionen von Kokain, von welchem je nach der Größe des Tieres 5 bis $10 \mathrm{ccm}$ einer I bis 5 proz. Lösung verabreicht wurde, das Schmerzgefühl nach kurzer Zeit erlosch, so daß weder Brennen der Haut mit dem Thermokauter, noch Zwicken der sonst empfindlichsten Körperpartien (Schnauze, Zunge, Pfoten, Scrotum usw.) mit Kocherklemmen überhaupt empfunden wurden. Auch die Oberfläche der Bauchhöhle und das Peritoncum parietale erwies sich als vollständig unempfindlich, ebenso das Betupfen ausgedehnter Darmpartien mit Chlorbarium-Lösungen, was sonst regelmäßig heftigste Schmerzen auslöste.

Meyer konnte durch seine Versuche (Ausschalten einer Extrcmität rom Blutkreislauf) sicher beweisen, daB es sich dabei um eine rein periphere Wirkung des Kokains handle und daß die größern Nervenstämme sich genau so empfindlich erwiesen wie vor der Injektion.

Kitter hat bei keinem der Tiere nach Kokaininjektion Er. brechen beobachtet, wohl aber regelmäßig nach Novokain. Alypin erwies sich als ungefährlicher als Kokain, aber nicht so sicher wirkend. Dagegen erreichte Stovain gleiche Wirkung wie Kokain bei größerer Ungefährlichkeit. Keines der Versuchstiere ist an den Folgen der Injektion gestorben, nur bei 3 von 50 Tieren konnte Ritter bei hohen Kokaindosen Krämpfe erzeugen.

Am Menschen wurden diese Versuche bis jetzt nicht gemacht, trotzdem bei Tierversuchen eine totale peripherische Analges i e erreichbar war. Ließen sich die Versuche auf den Menschen übertragen, so könnten auf solche Weise jene Operationen schmerzfrei ausgeführt werden, bei denen nicht größere Nervenstämme das Operationsgebiet kreuzen, z. B. sämtliche Eingriffe in der Bauchhöhle bei medianem Laparotomieschnitt.

Bei unseren zwei Fällen von akuter Novokainvergiftung wurde leider - wohl infolge der alarmierenden Symptome - unterlassen, auf diese periphere Analgesie zu prüfen. Auffallende Schlafzustände konnten wir nicht beobachten. Unsere zwei Beobachtungen scheinen mir insofern aber wertvoll, als sie zeigen, daß trotz großen Mengen Novokain intravenös injiziert $(0,4$ und $0,5 \mathrm{~g}$ Novokain in Substanz) und trotz den schweren plötzlichen Kollapserscheinungen, die Intoxikation ohne bleibenden Schaden für die Patienten ablaufen kann. 
Die ernsten dabei a uftretenden $Z$ ufälle lehren uns immerhin, daB die Injektionsnadeln, wenn sie einmal liegen, und die Kontrollaspiration erfolgt ist, a chnicht mehrum Bruchteilevon Millimetern verschoben werden dürfen. Deshalb ist Vorsichtbeim Aufsetzen der Spritze geboten und absolute Ruhiglage des Patienten während der In jektion. Wir pflegen deshalb unsere Kranken vorher genau über die ev. auftretenden Schmerzen aufzuklären, die übrigens individuell sehr verschieden empfunden werden. Außerdem unterhält sich die Krankenschwester zur Ablenkung mit dem Patienten während des Einführens der Nadeln und der Vornahme der Injektionen.

Die Dauer der Anästhesie schwankte zwischen $21 / 2$ und mehreren Stunden. Wir hatten den Eindruck, daß da, wo häufige Manipulationen in der Nähe der Depots durch den Gang der Operation nötig wurden, sich die anästhesierende Wirkung weit rascher erschöpfte als bei kurzen Eingriffen, wo der Ort des Handelns fern von den Injektionen lag. Für die Erklärung dieser Beobachtung glaubten wir rein mechanische Momente, wie dauernde Palpation, häufiger Zug an den Mesenterien usw. in der Nähe der Depots und dadurch bedingte raschere Resorption des Novokains annehmen zu dürfen. Oft hielt die anästhesierende Wirkung, vor allem nach kleineren Eingriffen, noch mehrere Stunden über den Schluß der Operation hinaus an, selbst bei Patienten, denen vorher keinerlei Narkotika verabreicht worden waren.

Wir haben bis heute I 50 Fälle in Splanchnicusanästhesie operiert, welche sich für die verschiedenen Eingriffe wie folgt verteilen:

Art der Operation

1. Appendektomien

2. Magenresektionen

3. Gastroenterostomien

4. Gastrostomien

5. Explorativ- u. Probelaparotomien 16

6. Dünndarmresektionen

7. Ileocöcalrescktionen
Anzahl Erg:ïnzende Mo. ante

d. Fälle Narkosen operationem

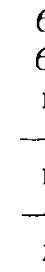

35

10

4

5

I

I 
Art der Operation Anzahl Ergänzende Mo, ante

d. Fälle Narkosen operationem

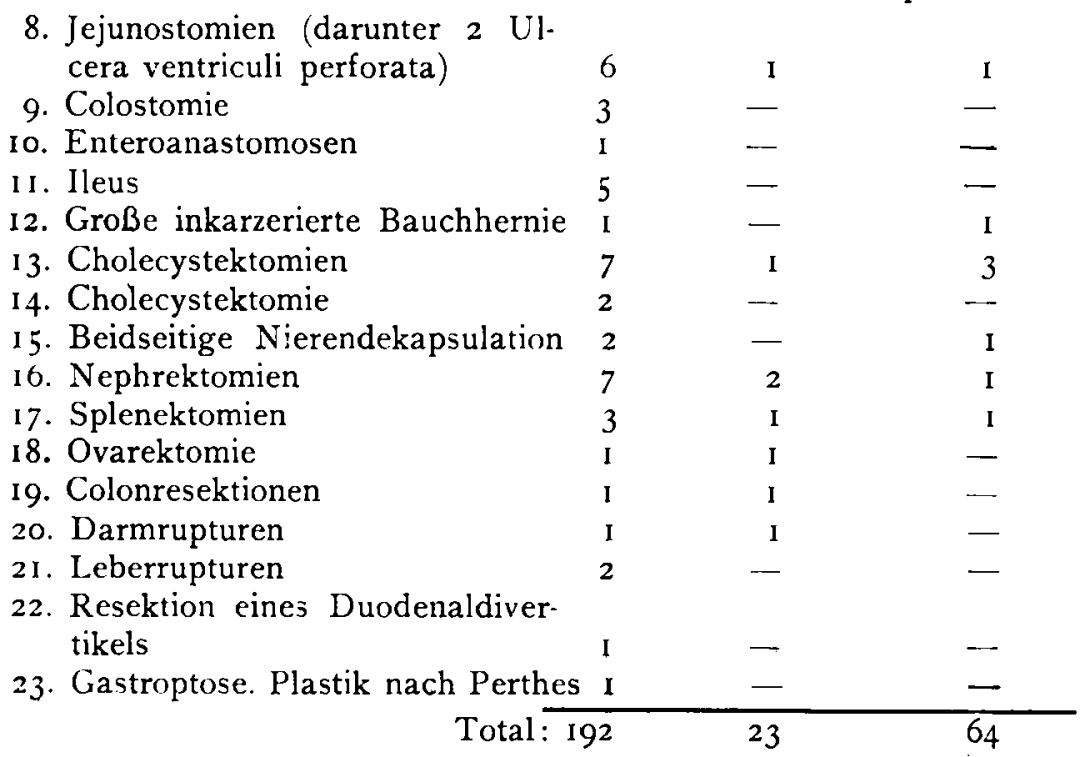

Die ergänzenden kurzen Narkosen, die in einigen Fällen angewendet werden mußten, waren bei länger dauernden Operationen wenigstens teilweise durch den Umstand bedingt, da $B$ die Kranken beim Schluß der Bauchhöhle und vor allem bei der Naht des parietalen Peritoneums zu pressen anfingen, also zu einer Zeit, wo dic Bauchdeckenanästhesie bisweilen schon ihre Wirkung verloren hatte und daher nicht gezögert wurde, auch diesen letzten $\mathrm{Akt}$ durch einen kurzen Ätherrausch schmerzlos zu gestalten.

Die älteste unserer Patienten, die in Splanchnicusanästhesie operiert wurde, betraf eine 74jährige Frau mit einer Chole- und Pericholecystitis, die jüngste ein 6jähriges Mädchen mit Ileus und subphrenischem Abszeß, bei welchem für Splanchnicus- und Bauchdeckenanästhesie im ganzen $0,45 \mathrm{~g}$ Novokain injiziert wurden. In beiden Fällen konnte der Eingriff bis zum Schluß ohne Narkose durchgeführt werden.

Der gute postoperative Verlauf ist bei der überwiegenden Mehrzahl der Patienten besonders auffallend und viele Kranke machten, selbst nach größeren Eingriffen, den Eindruck, als ob überhaupt nichts mit ihnen geschehen wäre. 
Wir glauben deshalb auf Grund der Erfahrungen mit der Splanchnicusanästhesie an der Züricher Klinik, die Methode mit gutem Gewissen empfehlen zu dürfen, ohne dabei zu gla u. ben, daB sie berufen wäre, die Narkose vollständig aus der Bauchchirurgie zu verdrängen. Neben der persönlichen Liebhaberei, die hier eine große Rolle spielen mag, gibt es aber doch häufig Kranke, denen auch der überzeugte Gegner der Lokalanästhesie keine Allgemeinnarkose mehr zumuten würde und die er deshalb einfach ihrem Schicksal überlassen müßte. Sieht man aber solch schwere Patienten nach Eingriffen in lokaler Anästhesie wieder gesund werden, so hat man gewiß ein Recht, an die Zuverlässigkeit und die Zukunft eines Verfahrens zu glauben, das bei vorsichtiger Technik schon jetzt vermutlich nicht größere Gefahren in sich birgt als die Allgemeinnarkose.

\section{Zusammenfassung.}

I. In den sympathischen Nerven verlaufen neben den motorischen Neuronen auch sensible Fasern, von denen es nicht sicher ist, ob sie überhaupt sympathische Elemente sind.

2. Nach Langley besteht die zentripetale Bahn von der Peripherie bis zur Medulla spinalis aus einem einzigen Neuron, dessen trophisches Zentrum im Ganglion spinale liegt. Andere Autoren nehmen für die zentripetale Leitung mindestens 2 oder mehr Neurone an wie für die zentrifugale.

3. Für die sensible Schmerzleitung aus der Bauchhöhle kommen nur in Betracht:

I. die Nervi splanchnici,

2. die von den lumbalen Grenzstrangganglien peripherwärts ziehenden Fasern.

Über den Vagus wird keine Schmerzempfindung aus der Bauchhöhle zentripetal geleitet.

4. Nach unseren Befunden ist eine strenge Metamerie der Ganglien des lumbalen Grenzstrangs selten. In den meisten Fällen wird eine Verschmelzung derselben in einen mehr oder weniger großen Ganglienknoten gefunden, der meist in der Höhe des 3. Lendenwirbels liegt. 
5. Wir schlagen deshalb vor, die 2 kaudal gelegenen Injektionsstellen des ursprünglichen $\mathrm{Kapp}$ is schen Verfahrens in die Höhe des 3. Lendenwirbels zu legen und empfehlen diese Modifikation namentlich für Eingriffe, die Nieren, Milz und C.olon betreffen.

6. Das Verfahren der Injektion von vorn nach eröffneter Bauchhöhle kann bei der eigentlichen Kappisschen Splanchnicusanästhesie ergänzend in Form von sekundären Injektionen angewendet werden.

7. Die besten Resultate haben wir bis heute erhalten bei Verwendung einer 2 proz. Novokain-Suprarenin-Lösung mit Zusatz von Kalium sulfuricum.

8. Das Anstechen von Gefäßen ist belanglos, nicht aber die Injektion des Anästhetikums in dieselben.

9. Die akuten Novokainvergiftungen äußern sich in Form schwerer Kollapse mit Krampfanfällen und Atemstillstand. Bleibende Schädigungen haben wir selbst nicht beobachtet.

\section{Literaturverzeichnis.}

I. B i ed1, Über die Centra der Splanchnici. Wiener klin. Wochenschr. 1895, Nr. 52, S. 915.

2. -, Innere Sekretion. Wien 1913.

3. Bier, Uber den heutigen Stand der Lumbal- und Lokalanästhesie. Deutscher Chir.Kongr. 1909, Bd. II, S. 474.

4. B i er, Bakes, Bemerkung zur Sensibilität der Bauchorgane. Deut. scher Chir.-Kongr. 1909, Bd. I, S. 26I.

5. Bircher, Die operative Behandlung der Ulcuskrankheit. Schweiz. Rundschau f. Medizin 1918, H. 24-26, S. 44.

6. Braun, Die örtliche Betäubung, 19I9, S. $320 \mathrm{ff}$. u. 354 ff., mit ausführlichen Literaturangaben.

7. Bra un e. Topographisch-anatomischer Atlas 1875.

8. Bruce, Distribution of the cells in the intermedio-lateral tract of the spinal cord. Transact. of the Royal Soc. of Edinburgh 1906, vol. 45, zit. nach Müller.

9. Buch, Die Sensibilitätsverhältnisse des Sympathicus und Vagus mit besonderer Berücksichtigung ihrer Schmerzempfindlichkeit im Bereiche der Bauchhöhle. Engelmanns Arch. f. Physiol. 1901, S. 197. 
10. Buerger und $\mathrm{Churchmann}$, Der Plexus coeliacus und mesen. tericus und ihre Rolle beim Abdominalshok. Mitteilungen a. d. Grenzgeb. I906, Bd. XVI, S. 507 .

II. B uhre, Die Leitungsanästhesie bei Operationen in der Bauchhöhle und die Unterbrechung der Nervi splanchnici. Bruns' Beitr. 1919, 13d. I18, S. 5 I.

12. -, Die Unterbrechung der Nervi splanchnici bei Bauchoperationen. Deutsche med. Wochenschr. 1920, H. 4, S. 93.

13. Cajal, Sur les ganglions et les plexus nerveux de l'intestin. Comptes rend. $1893 / 94$, Bd. 4 , S. 217.

14. -, Système nerveux des vertébrés I9I I.

15. Castellino e Pendo, Patologia del simpatico. Milano 1915.

16. Chalier, Chirurgie du sympathique. Paris $191 \mathrm{I}$.

17. Denk, Erfahrungen mit der Kappisschen Splanchnicusanästhesie. Wien. klin. Wochenschr. 1919, Nr.41, S. 999.

18. Dogiel, Zur Frage über den feineren Bau des sympathischen Ner. vensystems bei den Säugetieren. Arch. f. mikroskop. Anatomie 1895, Bd. 46, S. 305 .

19. Dr ü ner, Uber Bauchoperationen in örtlicher Betäubung. Bruns' Beiträge 1919, Bd. I 18, S. 222.

20. F inst e rer, Lokalanästhesie bei Magenoperationen. Zentralbl. f. Chir. IgI I, S. I 504 .

2I. - Zur Technik der paravertebralen Leitungsanästhesie. Zentralbl. f. Chir. 1912, S. 601.

22. - Lokalanästhesie bei Magenoperationen. Bruns' Beiträge I9I2, Bd. 8I, S. 266.

23. Fröhlich und Mejer, Die sensible Innervation von Darm und Harnblase. Wiener klin. Wochenschr. I9I2, H. I, S. 29.

24. Gaskell, The involuntary nervous system. IgI6.

25. Gegenbaur. Lehrbuch der Anatomie des Menschen igio.

26. Goldscheider, Zur Frage der Schmerzempfindlichkeit des visceralen Sympathicusgebiets. Deutsche Zeitschr. f. Chir. 1908, Bd. 95, S. 1.

27. Got ts te in, Lokale Anästhesie an der Breslauer Klinik. Arch. f. klin. Chir. 1898, Bd. 57, S. 409.

28. - Die gleichzeitige, doppelseitige Vagotomie und ihr Einfluß auf die Cardia. Breslau 1902.

29. Hackenbruch, Örtliche Schmerzverbütung bei Bauchoperationen. Deutsche Zeitschr. f. Chir. 1914, Bd. I29, S. 168.

3o. Hacker, v., Zur Lokalanästhesie. Wiener klin. Wochenschr. 1893, Nr. 10, S. 174 .

31. Härte 1, Die Lokalanästhesie. Neue deutsche Chir. 1916, Bd. 21.

32. H e ad, Sensibilitätsstörungen der Haut bei Visceralerkrankungen. Deutsch von Seiffer. Berlin 1898 .

33. Hesse, Inwieweit vermag bereits jetzt die Lokalanästhesie die allgemeine Narkose zu ersetzen. Deutsche med. Wochenschr. I910, Nr.8, S. 359 . 
34. Ifesse, Die örtliche Schmerzrerhïtung in der Chirurgie. Würzburger Abhandl. I9lo.

35. Hirschel, Fortschritte auf dem Gebiete der Lokalanästhesie. Mcd Klinik IgII, S. 1721 .

36. Hirsch feld und Leveillé. Nérrologie ou description et iconographie du système nerveux et des organes des sens de l'homme. Paris 1853.

37. H of $\mathrm{mann}$ und $\mathrm{Kochmann}$, Untersuchungen über die Kombi. nation der Lokalanästhetica mit Kaliumsulfat, nebst Angabe einer einfachen Wertbestimmungsmethode. Bruns' Beiträge I9I 4, Bd.9I, S. 489 .

38. - - Verminderung der Novokainkonzentration durch Kaliumsulfat bei der Lokalanästhesie. Deutsche mcd. Wochenschr. 1912, Nr. 48, S. 2264 .

39. H of $\mathrm{fmann} \mathrm{n}$, Splanchnicusanästhesie in der Bauchchirurg.e. Zentralbl. f. Chir. 1920, Nr. 3, S. 54 .

40. Hotz, Beiträge zur Pathologie der Darmbewegungen. Mitteil. a. d. Grenzgeb., Bd. 20. S. 257.

4I. J a b o u la y, Chirurgie du sympathique et du corps tmyroide. Paris 1920.

42. -, Chirurgie des centres nerveux, des viscères et des membres. Paris igo2.

43. Jentzer, Les points de repère anatomiques et les résultats de l'anesthésie paravertébrale. Rev. méd. de la Suisse rom. 1919, S. 271.

44. I hring, Die nervöse Dyspepsie. Volkmanns Vortr. Nr. 283.

45. Jonesco, Die allgemeine Rachianästhesie. Deutsche med. Wochenschr. 1912, Nr. 19, S. 905.

46. Jurasz, Die paravertebrale Anästhesie im Dienste der Gallensteinchirurgie. Zentralbl. f. Chir. 1914, S. 1409.

47. Kappis, Leitungsanästhesie am Bauch, Brust, Arm, Hals durch Injektionen ins Foramen intervertebrale. Münch. med. Wcchenschr. 19 r2. Nr. I 5, S. 794.

48. --, Über Leitungsanästhesie bei Nierenoperationen und Thorakoplastiken, überhaupt bei Operationen am Rumpf. Zentralbl. f. Chir. Igı2. S. 249.

49. -, Zur Frage der Sensibilität der Bauchorgane. Mitteil. a. d. Grenzgeb. 1913, Bd. 26, S. 493.

50. --, Erfahrungen mit Lokalanästhesie bei Bauchoperationen. Verhandl. d. Deutschen Ges. f. Chir. I914, 43. Kongr., S. 87.

51. -, Die Anästhesie des Nervus splanchnicus. Zentralbl. f. Chir. I9I8. Nr. 40, S. 7c9.

52, - Sensibilität und Lokalan̈ïsthesie im chirurg. Gebiet der Bauchhöhle mit besonderer Berücksichtigung der Splanchnicusanästhesie. Bruns' Beiträge 1919, Bd. I I5, S. I6I.

53. -, Zur Technik der Splanchnicusanästhesie. Zentralbl. f. Chir. I920, Nr. 5, S. 98 .

54. Kast, Zur theoretischen und praktischen Bedeutung der $\mathrm{H}$ e a dschen Zonen bei Erkrankungen der Verdauungsorgane. Berliner klin. Wochenschr. 1906, Nr. 31, S. 1033, Nr. 32, S. 1070.

Deutsche Zeitschrift f. Chirurgie, 159. Bd. 
55. Kast und Meltzer, Sensibilität der Abdominalorgane und die Beeinflussung derselben durch Cocain. Berliner klin. Wochenschr. 1907. H. I9, S. 600 .

56. - , Die Sensibilität der Bauchhöhle. Mitteil. a. d. Grenzgeb. 1909, Bd. 19, S. 586.

57. Kocher, Verletzungen der Wirbelsäule, zugleich als Beitrag zur Physiologie des menschlichen Rückenmarks. Die Läsionen des Rückenmarks bei Verletzungen der Wirbelsäule. Mitteil. a. d. Grenzgeb. 1896, Bd. I, S. $4 \mathrm{I} 5$ und $48 \mathrm{I}$.

58. $\mathrm{K}$ ölliker, liber die feinere Anatomie und physiolog. Bedeutung des sy'mpathischen Nervensystems. Münchner med. Wochenschr. 1894, S. 812.

59. Laignel-Levastine, Recherches sur le plexus solaire. Paris 1903 .

6o. Langley, The sympathetic and other related systems of nerves. Schäfers Textbook of Physiologie 1900, vol. 2, p. 616.

61. -, On axon-reflexes in the preganglionic fibres of the sympathet:c system. Journ. of Physiol. 1900, vol. 25, p. 364.

62. - Das sympathische und verwandte nervöse Sistem der Wirbeltiere (autonomes nervöses System). Asher-Spiro Ergebnisse d. Physiol. 1903, Bd. 2, S. 818 mit zahlreichen Literaturangaben.

63. Langley und Magnus, Some observations of the movements of the intestine before and after degenerative section of the mesenteric nerves. Journ. of Physiol. 1905, vol. 33, p. 47.

64. Langley und Anderson, On reflex action from sympathetic ganglion. Journ. of Physiol. 1894 , vol. 16, p. 410.

65. - - On the innervation of the pelvic and adjoining viscera. Journ. of Physiol. 1895, vol. I8, p. 82.

66. L a ngley und $\mathrm{Orbeli}$, The sympathic innervation of viscera. Journ. of Physiol. 1910, vol. 40.

67. - - Some observations on the degeneration in the sympathetic and sacral autonomic nervous system of amphibia following nerve section. Journ. of Physiol. 1912, vol. 42, p. 113 .

68. Läwen, Lokalanästhesie bei Nierenoperationen. Münchner med. Wochenschr. I911, H. 26, S. 1390.

69. -, Die Verbindung der Lokalanästhesie mit der Narkcse, über hohe Extraduralanästhesie und epidurale Injektionen anästhesierender Lösungen bei tabischen Magenkrisen. Bruns' Beiträge I912, Bd. 80, S. I68.

70. Lennander, Sensibilität der Bauchhöhle, lokale und allgemeine Anästhesie bei Bruch- und Bauchoperationen. Zentralbl. f. Chir. 19or, S. 209 .

71. -, Beobachtungen über die Sensibilität in der Bauchhöhle. Mitteil. a. d. Grenzgeb. 1902, Bd. 10, S. 38.

72. -, Weitere Beobachtungen über Sensibilität in Organ und Gewebe und über lokale Anästhesie. Deutsche Zeitschr. f. Chir. I904. Bd. 7.3. S. 297 . 
73. L, en nander, Lokale Anästhesie und Sensibilität in Organ und Gewebe. Mitteil. a. d. Grenzgeb. 1906, Bd. 15, S. 465.

74. - U Uber Hofrat Nothnagels zweite Hypothese der Darmkolikschmerzen. Mitteil. a. d. Grenzgeb. I906, Bd. I6, S. 19.

75. - Leibschmerzen, ein Versuch einige von ihnen zu erklären. Mitteil. a. d. Grenzgeb. 1906, Bd. 16, S. 24.

76. Loder. Tabulae anatomicae 1803 .

77. Luciani. Tratto di fisiologia. Milano.

78. M e umann, Zur Frage der Sensibilität der inneren Organe. Arch. f. d. ges. Psychol. 1907, Bd. 9, S. 26 und 1909, Bd. I4, S. 279.

79. M e yer, Experimentelle Untersuchungen über die Sensibilität von Magen und Darm. Deutsche Zeitschr. f. Chir. I919, Bd. I5I, S. 15I.

8o. Meyer und Gottlieb. Experimentelle Pharmakologie Igr4.

81. M ichailow, Der Bau der zentralen sympath. Ganglien. Internat. Monatsschr. f. Anat. u. Physiol. 19II, Bd. 28, S. 26, mit zahlreichen Literaturangaben.

82. Mikulicz, Methoden der Schmerzbetäubung und ihre gegense.tige Abgrenzung. Arch. f. klin. Chir. I90I, Bd. 64, S. 757.

83. M üller, L. R., Die Empfindung in unseren inneren Organen. M.t. teil. a. d. Grenzgeb. 1908, Bd. 18, S. 6=0.

84. - Studien über die Anatomie und Histologie des sympatischen Grenzstrangs, insbesondere über seine Beziehungen zu dem spinalen Nerven. system. Verhandl. d. 26. Kongr. f. inn. Med. Bergmann, Wiesbaden 1909.

85. - Allgemeine Bemerkungen zur Physiologie des vegetativen Nervensystems. Deutsche med. Wochenschr. 19i , Nr. 13, S. $5^{83}$.

86. -, Darminnervation. Arch. f. klin. Med. I9II, Bd. I05, S. I.

87. Muroya, Experimentelle Untersuchungen über Novokain bei Paravertebralinjektionen. Deutsche Zeitschr. f. Chir. 1913, Bd. I22, 6. I.

88. Na egeli, Splanchnicusanästhesie bei chirurgischen Eingriffen und schmerzhaften Affektionen in der Bauchhöhle. Zentralbl. f. Chir. 1919, S. 749 .

89. Nast-Kolb, Beitrag zur Frage der Sensibiliät der Bauchorgane. Zentralbl. f. Chir. 1908, S. 857.

90. $\mathrm{N}$ eumann, Uber die Sensibilität der inneren Organe. Zentralbl. f. d. Grenzgeb. I910, Bd. 13, S. 40I.

91. -, Zur Frage der Sensibilität der inneren Organe. Zentralbl. f. Physiol. 1910, Bd. 24, S. 1210.

92. - Desgleichen: Funktioneller Nachweis sensib'er Fasern im Splanchnicus und Vagus. Zentralbi. f. Physiol. I910, Bd. 24, S. I213.

93. - Desgleichen: Sensible Reizung entlang dem Darm. Zentralbl. f. Physiol. 1910, Bd. 24, S. 217.

94. -, Desgleichen: Ein Beitrag zur Funktion des Plexus myentericus. Zentralbl. f. Physiol. 19II, Bd. 25, S. 53.

95. - Desgleichen: Über schmeraleitende Fasern im Nervus splanchnicus und Grenzstrang des Hundes. Zentralbl. f. Physiol. I9ı2, Bd. 26, S. 277. 
95. N ot hn ag e 1, Zur Pathogenese der Kolik. Arch. f. Verdanungskrankheiten I905, Bd. I I, S. I 7 .

97. Onodi, Über die Entwicklung des sympathischen Nervensystems. Arch. f. mikroskop. Anatomie 1886, Bd. 26, S. 6I u. 553.

93. O nuf and Collins, Experimental researches on the central localisations of the sympathetic with a critical revue of its anatomy and physiology: Arch. of Neurology and Psychopathology 19oo, vol. 3, p. I.

99. I'fanner, Zur Frage der Lokalananästhesie in d:r Rauchchirurg.c. Wiener klin. Wochenschr. 1918, S. 863.

100. Potter, Topography of the thorax and abdomen, 1905.

Ior. P oulsson. Lehrbuch der Pharnakologie 1912.

102. Prei $B$ und $R$ itter, Zur Anwendung der Splanchnicusanästhesie. Zen. tralbl. f. Chir. 1919, Nr. 52, S. 1025.

I03. Propping, Zur Frage der Sensibilität der Bauchhöhle. Bruns' Be:träge 1909, Bd. 63, S. 690 .

104. Ramström, Untersuchungen und Studien über dis Innervation des Peritoneums der vorderen Bauchwand. Anatom. Hefte 19I5, Bd. 29, S. 89.

I05. Rauber-Kobsch. Lehrbuch der Anatomie 1914.

106. Rein har d, Die Vorzüge der Novokainleitungsanästhesie vor der Inhalationsnarkose. Erfahrungen a. d. Geb. d. Novokain-Leitungsanästhesie. Deutsche Zeitschr. f. Chir. 1917, Bd. 139, S. 110.

107. R itter, Zur Frage der Sensibilität der Eauchorgane. 7entralbl. f Chir. 1908, S. 609 .

108. -, Experimentelle Untersuchungen über die Sensibilität der Bauchhöhle. Arch. f. klin. Chir. 1909, Bd. 90, S. 389.

1c9. R obinson, The abdominal and pelvic Brain. 1907.

I Io. Ry nberk, va n, Metemaria simpatica. Mem. dell' acadımia med. di Roma I908.

111. Sa p pey. Anatomie humaine 1873 .

112. Schlimpert, Hohe und tiefe extradurale Anästhesien. Zentralbl. f. Gynäkol. I9I I, Bd. 12, S. 477.

113. Schmidt, Ein Beitrag zur Frage der Magensensibilität. Mitteil. a. d. Grenzgeb. 1909, Bd. I9, S. 278.

114. Schultz, Das sympathische Nervensystem. Nagels Handb. d. Physiol. 19=9, Bd. 4, S. $393 \mathrm{ff}$, mit zahlreichen Literaturangaben.

115. Schumacher, Zur Technik der Lokalanästhesie bei Thorakoplastiken. Zentralbl. f. Chir. 1912, S. 252.

116. Sellheim, Die Herabsetzung der Empfindlichkeit der Bauchdecken und des Peritoneum parietale durch perineurale Injektion anästhes:erender Lösungen an die Stämme der Nervi intercostales, des subcostalis, des iliohypogastricus und des ilioinguinalis. Verhandl. d. 'Deutsch. Ges. f. Gynäkolog. 1906, S. 176 .

117. Siegel, Die paravertebrale Leitungsanästhesie. Deutsche med. Wochenschr. 1914 , Nr. 28 , S. 1416.

118. -- Grundlagen und Technik der parasakralen Leitungsanästhesie. Zeitschrift f. Geburtsh. 4. Gynäkol. 1916, Bd. 79, S. 76. 
119. Si eg e 1, Ergebnisse bei weitcren 600 paravcrtebralen Anästhesien. Med. Klinik 1916, Nr. 2, S. 34 .

120. - Die paravertebrale I,eitungsanästhesic, ein vollwertiger Eisiltz der Inhalationsnarkose. Zentralbl. f. Gynäkol. 1917, Nr. 5, S. 121.

121. Sobotta. Anatomischer Atlas.

122. Spalt e holz. Handatlas der Anatomie 1909 .

123. $\mathrm{S}$ p isharny, Lokale Anästhesic. Ref. in Zentralbl. f. Chir. 190\%, S. 688 .

124. T'estut. Traité d'anatomie humaine 1905

125. Tigerstedt. Lehrbuch der Physiologie des Menschen 1909, Bd, 2.

126. Toldt. Anatomischer Atlas. Urban \& Schwa:zenberg, 1900.

127. Villiger, Die periphere Innervation. I9I9.

128. Vogt, Uber die Folgen der Durchschneidung des Nervus splanch. nicus. Arch. f. Anat. u. Physiol. 1 898, S. 399.

129. Wendling, Ausschaltung der Nervi splanchnici durch Lcitungsanästhesie bei Magenoperationen und anderen Eingriffen in der oberen Bauchhöhle. Burns' Beitr. 1918, Bd. I Io, S. 517.

130. Wilms, Die Ursachen der Kolikschmerzen bei Damleiden, Gallenund Nierensteinen. Nünchner med. Wcchenschr. 1904, S. 1377.

I3I. -, Zur Pathogenese der Kolikschmerzen. Mitteil. a. d. Grenzgeb. 1906, Bd. 16, S. 609 .

132. - Sensibilität und Schmerzempindung der Bauchorgane. Deutsclese Zeitschr. f. Chir. 1909, Bd. 100, S. 372 u. 512.

I33. -, Dic Schmerzempfindung innerhalb der Bauchhöhle und ihre Bedeutung für die Diagnose. Med. Klinik igri, S. I3.

134. Z we if el, Die Todesfälle bei Sakralanästhesie. Zcntralbl. f. Gynïkologie 1920, H. 6, S. 140.

135. B a kes, Beiträge zur Bauchchirurgie. Arch. f. klin. Chir. 1905, Bd. 74, S. 967 .

136. Friedländer, Erfahrungen über die Lokalanästhesic nach Schleich. Wiener klin. Wochenschr. I900, S. 1166.

137. Mackenzie, Krankheitszeichen und ihre Auslegung. 1917.

138. M e yer, A., Uber die sogenannte Totalanästhesie nach intravenöser Injektion von Lokalanästheticis. Arch. f. klin. Chir. 1914, Bd. I05, S. 170.

139. -- Über Intoxikationserscheinungen (Schlafzustand, Krämpfe, peripherische Totalanästhesie) nach Novokain-Lokalanästhesie be:m Menschen. Deutsche med. Wochenschr. 1919, S. 681.

140. Pawlow, Die Arbeit der Verdauungsdrüsen. Bergmann, Wiesbaden 1898 .

141. Ritter, Totalanästhesie durch Injektion ron Cocain in die Vene. Berliner klin. Wochenschr. 1919. S. I70r.

I42. -, Totalanästhesie durch Injckt:on von Lokalanästhet:cis ins B!ut. Arch. f. klin. Chir. 1911, Bd. 96, S. 928.

143. Schleich, Schmerzlose Operationen. Springer, Berlin 1894 